\title{
VEGAS-SSS. II. Comparing the globular cluster systems in NGC 3115 and NGC 1399 using VEGAS and FDS survey data
}

\section{The quest for a common genetic heritage of globular cluster systems ${ }^{\star}$}

\author{
Michele Cantiello ${ }^{1}$, Raffaele D’Abrusco ${ }^{2}$, Marilena Spavone ${ }^{3}$, Maurizio Paolillo ${ }^{4}$, Massimo Capaccioli ${ }^{4}$, \\ Luca Limatola $^{3}$, Aniello Grado ${ }^{3}$, Enrica Iodice ${ }^{3}$, Gabriella Raimondo $^{1}$, Nicola Napolitano ${ }^{3}$, John P. Blakeslee ${ }^{5}$, \\ Enzo Brocato ${ }^{6}$, Duncan A. Forbes ${ }^{7}$, Michael Hilker ${ }^{8}$, Steffen Mieske ${ }^{9}$, Reynier Peletier ${ }^{10}$, \\ Glenn van de Ven ${ }^{11}$, and Pietro Schipani ${ }^{3}$ \\ ${ }^{1}$ INAF Osservatorio Astr. di Teramo, via Maggini, 64100 Teramo, Italy \\ e-mail: cantiello@oa-teramo.inaf.it \\ 2 Smithsonian Astrophysical Observatory, 60 Garden Street, 02138 Cambridge, MA, USA \\ 3 INAF Osservatorio Astr. di Capodimonte Napoli, Salita Moiariello, 80131 Napoli, Italy \\ 4 Dip. di Fisica, Universitá di Napoli Federico II, C.U. di Monte Sant'Angelo, via Cintia, 80126 Naples, Italy \\ 5 NRC Herzberg Astronomy \& Astrophysics, Victoria, BC V9E 2E7, Canada \\ ${ }^{6}$ INAF Osservatorio Astronomico di Roma, via di Frascati, 33, 00040 Monteporzio Catone, Italy \\ 7 Centre for Astrophysics \& Supercomputing, Swinburne University, Hawthorn, VIC 3122, Australia \\ 8 European Southern Observatory, Karl-Schwarzschild-Str. 2, 85748 Garching bei München, Germany \\ 9 European Southern Observatory, Alonso de Cordova 3107, Vitacura, Santiago, Chile \\ 10 Kapteyn Astronomical Institute, University of Groningen, PO Box 72, 9700 AV Groningen, The Netherlands \\ 11 Max Planck Institute for Astronomy, Königstuhl 17, 69117 Heidelberg, Germany
}

Received 17 February 2017 / Accepted 16 October 2017

\begin{abstract}
We analyze the globular cluster (GC) systems in two very different galaxies, NGC 3115 and NGC 1399. With the papers of this series, we aim at highlighting common and different properties in the GC systems in galaxies covering a wide range of parameter space. We compare the GCs in NGC 3115 and NGC 1399 as derived from the analysis of one square degree $u$-, $g$-, and $i$-band images taken with the VST telescope as part of the VST early-type galaxy survey (VEGAS) and Fornax deep survey (FDS). We selected GC candidates using as reference the morpho-photometric and color properties of confirmed GCs. The surface density maps of GCs in NGC 3115 reveal a morphology similar to the light profile of field stars; the same is true when blue and red GCs are taken separately. The GC maps for NGC 1399 are richer in structure and confirm the existence of an intra-cluster GC component. We confirm the presence of a spatial offset in the NGC 1399 GC centroid and find that the centroid of the GCs for NGC 3115 coincides well with the galaxy center. Both GC systems show unambiguous color bimodality in $(g-i)$ and $(u-i)$; the color-color relations of the two GC systems are slightly different with NGC 3115 appearing more linear than NGC 1399. The azimuthal average of the radial density profiles in both galaxies reveals a larger spatial extent for the total GCs population with respect to the galaxy surface brightness profile. For both galaxies, the red GCs have radial density profiles compatible with the galaxy light profile, while the radial profiles for blue GCs are shallower. As for the specific frequency of GCs, $S_{N}$, we find it is a factor of two higher in NGC 1399 than for NGC 3115 ; this is mainly the result of extra blue GCs. By inspecting the radial behavior of the specific frequency, $S_{N}(<r)$, for the total, blue, and red GCs, we find notable similarities between the trends for red GCs in the two targets. In spite of extremely different host environments, the red GCs in both cases appear closely linked to the light distribution of field stars. Blue GCs extend to larger galactocentric scales than red GCs, marking a significant difference between the two galaxies: the blue/red GCs and field stellar components of NGC 3115 appear well thermalized with each other and the blue GCs in NGC 1399 appear to fade into an unrelaxed intra-cluster GC population.
\end{abstract}

Key words. galaxies: elliptical and lenticular, cD - galaxies: star clusters: general - galaxies: individual: NGC 1399, NGC 3115 galaxies: clusters: individual: Fornax - galaxies: evolution - galaxies: stellar content

\section{Introduction}

Understanding the formation and evolution of galaxies is fundamental to shed light on the assembly of the baryonic matter

\footnotetext{
$\star$ The full catalogue of candidate sources is only available at the CDS via anonymous ftp to cdsarc. u-strasbg. fr (130.79.128.5) or via http://cdsarc.u-strasbg.fr/viz-bin/qcat?]/A+A/611/A93
}

in the Universe during the cosmic growth of large-scale structures. Although galaxies can be observed out to extremely large distances, the study of the properties of local galaxies remains fundamental. The redshift $z \sim 0$ is the current end point of galaxy evolution and an accurate comprehension of all the properties of local structures is essential for characterizing in detail the processes that lead to the present organization of matter (Mo et al. 2010). 
Globular clusters (GCs) are an important tool for understanding the formation and evolution of galaxies (Harris 2001; Brodie \& Strader 2006; Peng et al. 2008; Georgiev et al. 2010; Harris et al. 2013; Durrell et al. 2014).

Extragalactic, unresolved GCs are possibly the simplest class of astrophysical objects beyond stars. To a first approximation, GCs host a simple, single age, and single metallicity stellar population. However, the last decade has seen a growing amount of studies proving that the classical paradigm GCs $\equiv$ simple stellar populations is not valid for a large number of Milky Way (MW) GCs and for some of the clusters in the Magellanic Clouds (Gratton et al. 2004; Piotto et al. 2007; Carretta et al. 2009). Nevertheless, there is no doubt that GCs host a stellar population that is much simpler than galaxies in terms of metallicity and age distributions because GCs have a simpler star formation history.

Two other properties make GCs very useful: old age and high luminosity. In the few galaxies beyond the MW for which spectroscopic or multiband photometric studies of GCs have been carried out, the results almost uniformly pointed out a population with mean ages comparable to the GC system of the MW, older than 10 Gyr (e.g., Cohen et al. 1998, 2003; Strader et al. 2005; Chies-Santos et al. 2011). This makes GC systems the fossil tracer of the formation of a galaxy and its environment.

Moreover, extragalactic GCs appear as bright clumps of light on the otherwise smooth light profile of the galaxy and under typical observing conditions from the ground, they appear as point-like sources. The compactness and high contrast with respect to the background light from the galaxy and sky make GCs observable out to large distances. Photometric studies have been carried out for a GC system at $z \sim 0.2(d \sim 800 \mathrm{Mpc}$, HST/ACS data; Alamo-Martínez et al. 2013) and, more recently, at $z \sim 0.3(d \sim 1250 \mathrm{Mpc}$, HST/ACS, and WFC3 data; Janssens et al. 2017). The spectroscopic observations are much more limited and feasible only for the nearest, brightest GCs even with the largest $8-10 \mathrm{~m}$ class telescopes (Brodie et al. 2014).

The systematic study of GC systems (GCSs hereafter) in galaxies has highlighted a wealth of properties that are used to trace the physical characteristics of the GCS and its host galaxy; these characteristics include the luminosity function of GCs (GCLF), spatial distribution, projected surface density, radial color profiles, specific frequency, kinematical properties, and color-magnitude relations. All such properties are effective tracers of the past formation and evolution history of the galaxy, its physical distance, possible merging events, mass distribution, etc. (Harris 2001; Brodie \& Strader 2006).

In this paper we present and discuss the results from the analysis of the properties of the GCSs hosted by NGC 3115 and NGC 1399. The two galaxies analyzed are very different from each other and reside in dissimilar environments. We benefit from such extreme diversity to search for similarities and differences with the aim of providing useful constraints to separate intrinsic properties of GCSs, from the extrinsic properties in a process that will continue with the future studies of the VST elliptical galaxy survey (VEGAS) series on small stellar systems (VEGAS-SSS).

Table 1 summarizes the basic properties and observational details of the galaxies.

NGC 3115 is one of the closest lenticular galaxies, at $\sim 10 \mathrm{Mpc}$, located far south of its closest group of galaxies, the Leo I Group. The galaxy is very isolated; within a $2 \times 2 \mathrm{deg}^{2}$ area only one extragalactic source brighter than $m_{V} \sim 15 \mathrm{mag}$ and with $c z \leq 1500 \mathrm{~km} \mathrm{~s}^{-1}$ (i.e., $M_{V} \sim-15 \mathrm{mag}$ if at the distance of NGC 3115) can be found ${ }^{1}$. The closest source is the companion galaxy NGC 3115-DW01. The total number of extragalactic sources increases virtually to two objects within an area of $10 \times 10 \mathrm{deg}^{2}$, but the second angularly closest and bright object is Sextans A, a dwarf spheroidal galaxy in the Local Group, at $\sim 1.4 \mathrm{Mpc}$. Hence, on the $100 \mathrm{deg}^{2}$ area, only one bright galaxy is spatially close to NGC 3115 .

The GCS of NGC 3115 is perhaps the best case, beyond our Galaxy, to reveal a clear-cut bimodal color distribution that has been unambiguously demonstrated as due to a bimodal metallicity distribution. In this case both spectroscopic and (optical and near-IR) photometric studies consistently indicate the presence of a GC system with bimodal metallicity distribution with peaks at $[\mathrm{Fe} / \mathrm{H}] \sim-1.3$ and $\sim 0.0$ (Brodie et al. 2012; Cantiello et al. 2014).

The other target, NGC 1399, is classified as an E1 galaxy located near the dynamical center of the Fornax cluster and is the second brightest early-type galaxy of the cluster; the brightest is NGC 1316, which is offset by $\sim 3.5$ degrees southwest of the main body of the cluster (Ferguson 1989; Iodice et al. 2016, in prep.), although at nearly the same distance (Cantiello et al. 2013). The $2 \times 2 \mathrm{deg}^{2}$ region around NGC 1399 harbors $19 \mathrm{ob}-$ jects brighter than $m_{V} \sim 16.5 \mathrm{mag}$ and $c z \leq 3000 \mathrm{~km} \mathrm{~s}^{-1}$, which is equivalent to $M_{V} \sim-15 \mathrm{mag}$ if the source is at the adopted distance for NGC 1399; the number increases to 43 objects within a $10 \times 10 \mathrm{deg}^{2}$ area. Thus, NGC 1399 resides in a much denser galaxy environment than NGC 3115 .

The GCS of NGC 1399 is well studied in the literature with a great variety of studies from ground-based photometric and spectroscopic data (Geisler \& Forte 1990; Kissler-Patig et al. 1997; Forbes et al. 2001; Richtler et al. 2004; Bergond et al. 2007; Firth et al. 2007; Schuberth et al. 2008; Hilker 2015) and space-based X-ray, optical, and near-IR data (Forbes et al. 1998; Gebhardt \& Kissler-Patig 1999; Grillmair et al. 1999; Larsen et al. 2001; Kundu et al. 2005; Villegas et al. 2010; Mieske et al. 2010; Liu et al. 2011; Paolillo et al. 2011; Puzia et al. 2014; Jordán et al. 2015, in addition to the others cited elsewhere in this work).

NGC 1399 was one of the first cases of an early-type galaxy with the GCS revealing a pronounced bimodal color distribution in optical bands (Ostrov et al. 1998; Dirsch et al. 2003). A more recent study of the inner GC system in the galaxy, based on ACS optical and WFC3 near-IR data, has obtained observational evidence for the nonlinearity of its color-metallicity relations (Blakeslee et al. 2012). The study also revealed inconsistent properties of the color distributions between the optical and near-IR colors, such as different likelihoods of color bimodality and different red to blue GC ratios. Such inconsistency between optical and near-IR color distributions was one of the predictions proposed as evidence in support of the so-called projection scenario (Yoon et al. 2006; Cantiello \& Blakeslee 2007, which we discuss further later in this section). Unfortunately, despite many studies over the years, spectroscopic metallicities for a large sample of GCs in NGC 1399 are not available.

A further peculiarity of GCs in NGC 1399 is the observational evidence supporting the membership of a fraction of the GCs to the Fornax cluster rather to the galaxy itself (Grillmair et al. 1994; Kissler-Patig et al. 1998; Bassino et al. 2003; Schuberth et al. 2010; D’Abrusco et al. 2016). The presence of an intergalactic GC population allowed the resolution of another peculiarity of the GCS in the galaxy. The first studies on

1 Data from the NASA Extragalactic Database. 
the GC specific frequency ${ }^{2}$, obtained unusually high $S_{N}$ values (Harris \& Hanes 1987; Bridges et al. 1991; Wagner et al. 1991; Geisler \& Forte 1990; Ostrov et al. 1998). By associating a faction of GCs to the whole cluster and normalizing the specific frequency to the total light (galaxy plus halo), the estimated $S_{N}$ goes back to normal values for a system such as NGC 1399 and its environment (Peng et al. 2008; Georgiev et al. 2010).

Because of the isolation of NGC 3115 and the much denser cluster environment of NGC 1399, together with the presence of an intra-cluster GC component, our aim is to analyze the properties of the two GCSs and place their observed properties into a homogeneous and self-consistent picture to formulate useful constraints upon the importance of mergers and interactions on the global properties of the GC system.

Characterizing the GCS color bimodality in galaxies hosted in various environments, with different masses and morphologies, is one of the science cases for VEGAS-SSS. This science case has strong implications for GCS formation. Here we provide some background on the color (and metallicity) distribution properties of GC systems.

Color bimodality (CB hereafter) is a simple and yet dramatically important feature observed in nearly all galaxies massive enough to host a reasonable number of GCs $\left(N_{\mathrm{GC}} \gtrsim\right.$ 50). The GC bimodality consists of the presence of two well-defined peaks in color distribution separated by $\sim 0.2$ up to $\sim 1$ mag (Larsen et al. 2001; Kundu \& Whitmore 2001; Harris et al. 2006), depending mainly on the specific filters involved. Color bimodality is a very ubiquitous feature, is observed in very massive ellipticals near $M_{B} \sim-22$ mag (e.g., in M 87, the massive elliptical in Virgo), and in spheroidal galaxies some 5 mag fainter (Peng et al. 2006). This feature is found in ellipticals and spirals. It is found in galaxies over a range of environments from clusters and groups to isolated systems (Brodie \& Strader 2006; Peng et al. 2006).

Ashman \& Zepf (1992) initiated the interpretation that GC color bimodality was a consequence of GC metallicity bimodality. The idea was supported by the evidence that our bestknown reference galaxy, the MW, has a GC system with a bimodal $[\mathrm{Fe} / \mathrm{H}]$ distribution, where the metal-poor GCs (peak $[\mathrm{Fe} / \mathrm{H}] \sim-1.6)$ have a spatial distribution and kinematic properties associated with the Galaxy halo, and the metal-rich GCs (peak $[\mathrm{Fe} / \mathrm{H}] \sim-0.55$ ) are more centrally concentrated and show kinematic properties similar to bulge stars (Côté 1999). Moreover, as already mentioned, there is growing evidence based on the data collected from the 1990s and beyond that GCs are all nearly coeval and old ( $t>10$ Gyr; Puzia et al. 2004; Cohen et al. 1998, 2003; Strader et al. 2005; Chies-Santos et al. 2011). As a consequence of the nearly constant old age and the fact that at fixed metallicity integrated optical colors for ages older than $\sim 8$ Gyr do not vary significantly because of age ${ }^{3}$, the metallicity is left as the only parameter that can explain the observed CB.

Thus it was natural to equate $\mathrm{CB}$ with metallicity bimodality (FeB hereafter). Such equivalence has dramatic implications for the processes of formation and evolution of GC systems and their host galaxies. If the assumption is correct then the FeB

\footnotetext{
2 A parameter relating the galaxy and GCS properties, quantified as the number of GCs per unit galaxy luminosity $S_{N} \equiv N_{\mathrm{GC}} \times 10^{0.4\left(M_{V}+15\right)}$, where $N_{\mathrm{GC}}$ is the total number of clusters and $M_{V}$ is the total absolute visual magnitude of the galaxy (Harris \& van den Bergh 1981; Harris 1991).

3 For example, at fixed metallicity the change in $V-I$ color for a simple stellar population in the age interval $8-14 \mathrm{Gyr}$ is $\left|\Delta(V-I)_{(8-14 \mathrm{Gyr})}\right| \sim$ 0.05 (SPoT simple stellar population models; Raimondo et al. 2005; Raimondo 2009).
}

of GC systems is a very common feature and formation models need to account for the existence of two GC subpopulations with markedly different mean $[\mathrm{Fe} / \mathrm{H}]$, which formed either in two different epochs or with two different mechanisms, or a combination of both. The great success of FeB as a valid interpretation of $\mathrm{CB}$ comes also from the fact that, as already mentioned, it was predicted before it was a commonly observed feature (Ashman \& Zepf 1992).

Since the 1990s many authors proposed several other models for GCS formation accounting for the FeB. All models had their own ingredients, pros, and cons. All of the models can be basically associated with one of the following three classes:

- FeB forms as a consequence of the dissipative merging of gas-rich galaxies: blue GCs are pristine clusters hosted by the halos of the first galaxies. Red GCs form later, along with intense star formation due to galaxy mergers. In such a scenario, a $\sim 2$ Gyr age gap exists between blue and the red GC subpopulations (Ashman \& Zepf 1992).

- FeB from hierarchical growth: massive seed galaxies host intrinsically more metal-rich GC systems because their potential well was able to capture the metals produced by the first generations of stars more efficiently. The satellite, lower mass galaxies host a more metal poor GC system. As a consequence of dissipationless merging between a massive galaxy and its nearby lower mass companions, the system of red GCs is joined by more metal-poor, blue GCs (Beasley et al. 2002; Côté et al. 1998; Hilker et al. 1999). There is no age gap required between GCs in this class of models.

- FeB generated in situ: both blue and red GCs are indigenous to the galaxy, formed in two distinct phases of star formation, and emerge from gas of differing metallicity. The blue and metal-poor GCs formed at an early stage in the collapse of the protogalactic cloud. The red and metal-rich GCs formed out of more enriched gas, roughly contemporaneously with the galaxy stars. An age gap of 1-2 Gyr is expected between the two GC subpopulations (Forbes et al. 1997).

The proposed scenarios include predictions and interpretations of observed quantities; successfully explaining only some of the known properties of GC systems (Beasley et al. 2002; Kravtsov \& Gnedin 2005; Bekki et al. 2008; Griffen et al. 2010; Kruijssen 2014; Li \& Gnedin 2014). One common ingredient in all scenarios is the existence of two subpopulations of GCs with different mean metallicities. However, the colorto-metallicity bimodality equivalence has been challenged by Yoon et al. (2006). The Yoon et al. argument against FeB focuses on the linearity of the color-metallicity relations (CMR). If CMRs are close to linear, or such to a good approximation, then the shape of any distribution from one space, for example, in the color domain, is preserved when transferred into another space, for example, to [Fe/H].

Since 2006 an increasing amount of observational evidence has been collected showing that the CMRs for the colors mostly used for the study of extragalactic GCSs $(V-I, g-z$, and $B-I$ ) exhibit a degree of nonlinearity (Peng et al. 2006; Richtler 2006; Blakeslee et al. 2012; Yoon et al. 2011, 2013) ${ }^{4}$. Nonlinear CMRs might imply that the observed CBs have a completely different shape when translated into metallicity (Richtler 2006; Cantiello \& Blakeslee 2007; Blakeslee et al. 2010; Kim et al. 2013b). If the CMR is strongly nonlinear, then a fraction of

4 Earlier evidence of CMR nonlinearities was presented in Harris \& Harris (2002) and Cohen et al. (2003). 
the observed CBs could be explained by the so-called bimodality projection effect, in which the observed color distribution does not actually match with the properties of the progenitor $[\mathrm{Fe} / \mathrm{H}]$ distribution. Determining the fraction of real vs. projected FeB will likely provide a key constraint for galaxy and GCs formation models.

Recently, Harris et al. (2017), using HST/ACS data of BCG galaxies, showed that color bimodality for these large GC systems breaks down and becomes more complex. In their photometric study of GCs, for three out of five BCGs in the series of papers, the authors conclude that “... the imposition of a bimodal Gaussian numerical model ... begins to look increasingly arbitrary".

The best method to reveal real FeB in GC distributions, and whether they are ubiquitous, is to measure accurate spectroscopic metallicities for a large sample of GCs in individual galaxies. Unfortunately, this requires good signal-to-noise ratio and is very expensive in terms of telescope time. As an example, even though more than 500 GCs in NGC 1399 have published radial velocities (Schuberth et al. 2010), only a handful have published metallicities. The Schuberth et al. analysis reveals significantly different kinematics for blue and red subpopulations. In a study of NGC 1399 GCs by Kissler-Patig et al. (1998) with the Keck telescope, these authors measured spectroscopic metallicities for 18 GCs. A comparison with optical $V-I$ colors indicated a slightly nonlinear CMR relation. Their GC metallicity distribution hinted at two peaks with $[\mathrm{Fe} / \mathrm{H}] \sim-1.5$ and -0.5 . Hence, NGC 1399 GC system hints at metallicity bimodality, but a larger sample is required for more robust conclusions, as the sample of 18 GCs represents $\ll 1 \%$ of the entire GC population of $N_{\mathrm{GC}} \sim 6500$ (Dirsch et al. 2003; Bassino et al. 2006) ${ }^{5}$. Even adding the sample of $\sim 50$ bright ultra compact dwarfs (UCDs) and massive GCs with estimates from Hilker (2015), the total number of objects with known metallicity is $\lesssim 1 \%$ of the total population.

Claims of GC system FeB have been made for several other galaxies based on metallicity sensitive lines at optical wavelengths, for example, NGC 5128 (Beasley et al. 2008), NGC 4594 (Alves-Brito et al. 2011), NGC 4472 (Strader et al. 2007), and M 87 (Cohen et al. 1998). The interpretation of such claims, however, are still controversial (Blakeslee et al. 2010, 2012). Using the near-IR calcium triplet lines (CaT) Usher et al. (2012) examined the GC metallicity distributions of several galaxies from the SLUGGS survey. They concluded that six out of the eight galaxies with sufficient data revealed metallicity bimodality. However, the color-CaT relations were often somewhat nonlinear and the bimodality peaks appeared at different inferred metallicities. The presence of a third, intermediate metallicity subpopulation (e.g., NGC 4365) can complicate the interpretation. The case of NGC 3115, which is the nearest galaxy in their sample, revealed the best consistency between its color and CaT-based metallicity distributions.

Here we use VEGAS images to obtain photometric data of the GC systems around two very different host galaxies NGC 1399 and NGC 3115 . We focus on the color distributions, color-color relations, and spatial properties of their GC systems.

The paper is organized as follows: the next section describes the observations and the procedures for data reduction and for the selection of GC candidates. The analysis of the surface distribution of GC candidates, color distribution and color-color relations, radial density profiles, and local specific frequency are

\footnotetext{
5 A more recent estimate from the ACSFCS survey is $N_{\mathrm{GC}} \sim 10000 \pm$ 3000 (Liu et al., in prep.).
}

Table 1. Properties of the targets.

\begin{tabular}{lcc}
\hline \hline & NGC 3115 & NGC 1399 \\
\hline Gal. longitude $(l, \mathrm{deg})$ & 247.782502 & 236.716356 \\
Gal. latitude $(b, \mathrm{deg})$ & 36.780999 & -53.635774 \\
$c z\left(\mathrm{~km} \mathrm{~s}^{-1}\right)$ & $663 \pm 4$ & $1425 \pm 4$ \\
$(m-M)$ & $29.87 \pm 0.09$ & $31.51 \pm 0.03$ \\
$M_{V}^{\text {tot }}(\mathrm{mag})$ & -20.9 & -23.4 \\
Type & $\mathrm{S} 0$ & $\mathrm{E} 1$ \\
$E(B-V)$ & 0.078 & 0.013 \\
$R_{\text {eff }}(\operatorname{arcsec})$ & 57 & 49 \\
$T_{\text {type }}$ & $-2.9 \pm 0.6$ & $-4.6 \pm 0.5$ \\
$\sigma\left(\mathrm{km} \mathrm{s} \mathrm{s}^{-1}\right)$ & $259 \pm 3$ & $334 \pm 5$ \\
$M g_{2}(\mathrm{mag})$ & $0.288 \pm 0.002$ & $0.335 \pm 0.002$ \\
\hline \multicolumn{3}{c}{} \\
\hline Exp. t. $u(\mathrm{~s})$ & 14800 & 10200 \\
Exp. t. $g(\mathrm{~s})$ & 8675 & 6300 \\
Exp. t. $i(\mathrm{~s})$ & 6030 & 4960 \\
PSF $(u / g / i, \operatorname{arcsec})$ & $1.1 / 1.0 / 0.9$ & $1.3 / 1.2 / 1.1$ \\
\hline
\end{tabular}

Notes. For NGC 3115 we adopted the distance from Tonry et al. (2001), using the updated calibration zeropoint from Cantiello et al. (2013); the total $V$ magnitude is from de Vaucouleurs et al. (1991), obtained with $B-V \sim 1$ from the HyperLeda archive http://leda.univ-lyon1.fr/; the effective radius is from Capaccioli et al. (1987). For NGC 1399 we adopted the mean Fornax cluster distance from Blakeslee et al. (2009); the total magnitude is the $g$-band from Iodice et al. (2016), transformed to $V$ using $g-V \sim 0.5$ from Cook et al. (2014), derived assuming $B-V \sim 1$ from HyperLeda; the effective radius is from Iodice et al. (2016). The morphological type code $T_{\text {type }}$, velocity dispersion $\sigma$ and $M g_{2}$ are from HyperLeda, the remaining properties are taken from NED http://ned.ipac. caltech.edu

presented in Sect. 3. Section 4 provides a discussion of the results. In Sect. 5 we summarize our findings. Finally, Appendix A contains a list of several interesting objects, mostly in the field of NGC 3115.

\section{Observations, data reduction, and analysis}

\subsection{Observations and image processing}

The data used in this study are from two VST GTO surveys: VEGAS (P.Is: M. Capaccioli, E. Iodice), and the Fornax Deep Survey (FDS; P.Is: M. Capaccioli and R. Peletier). We analyzed ugi-band data of NGC 3115, from VEGAS, and of the $\sim 1$ sq. degree area centered on NGC 1399, a target common to both VEGAS and FDS.

A detailed description of the surveys and procedures adopted for data acquisition and reduction can be found in Grado et al. (2012), Capaccioli et al. (2015), and Iodice et al. (2016). Therefore, we only provide a brief overview here.

The data were processed with VST-tube (Grado et al. 2012), a pipeline specialized for the data reduction of VST-OmegaCAM executing pre-reduction (bias subtraction, flat normalization), illumination and (for the $i$-band) fringe corrections, photometric, and astrometric calibration. Unlike our previous study on compact stellar systems in NGC 3115 (Cantiello et al. 2015), based on only $g$ and $i$ data, here we did not apply any selection cuts to the input images adopted for the final mosaics of the two targets. A summary of the VST observations is provided in Table 1. For both galaxies we assumed Galactic extinction from the Schlafly \& Finkbeiner (2011) recalibration of the Schlegel et al. (1998) infrared-based dust maps. 
The VEGAS survey has been obtaining deep optical imaging of galaxies with heliocentric redshifts $c z \leq 4000 \mathrm{~km} \mathrm{~s}^{-1}$ and total magnitude $M_{B}^{\text {tot }} \leq-19.2$ mag to study the signatures of diffuse stellar components and of compact stellar systems, out to poorly constrained galactocentric radii. At completion, it is expected the survey will collect data for $\sim 100$ early-type galaxies across a range of environments and masses. To date, data for about 30 galaxies have been collected. The status of the survey is regularly updated ${ }^{6}$.

The FDS is a joint effort of the VST FOCUS (Fornax ultradeep survey, P.I: R. Peletier) and VEGAS surveys. Similar to VEGAS, the project plan is to obtain deep optical imaging data. The target is the Fornax cluster, which is the second closest galaxy cluster after Virgo, and the scientific objectives of the survey are numerous: the study of the galaxy luminosity function, derivation of galaxy scaling relations, determination of the properties of compact stellar systems (from GCs to UCDs and cEs), an accurate determination of distances and 3D geometry of the Fornax cluster, and analyses of diffuse stellar light and galaxy interactions, etc. Some first results based on FDS data have already been presented in Iodice et al. (2016) to study the diffuse stellar halo of the cluster core to very faint limits, and in D'Abrusco et al. (2016), where a first analysis of the clusterwide globular cluster population is described. The survey also includes several follow-up programs, such as the MUSE observations of NGC 1396 (Mentz et al. 2016) and the spectroscopic follow-up of 2000 compact sources (observations carried out with VIMOS; Pota et al., in prep.).

Images were calibrated in the SDSS photometric system using several Landolt (1992) standard fields with calibrated SDSS photometry. By comparison with the literature data available for the $g$ and $i$ bands, we found median differences below $\sim 0.05 \mathrm{mag}$ with $\leq 0.05 \mathrm{mag} \mathrm{rms}$ in available bands - the data for NGC 3115 are from the catalogs of Jennings et al. (2014) and, for NGC 1399 GCs, the $g$-band photometry is from Jordán et al. (2015). The median offset did not show any trend with color.

\subsection{Galaxy modeling and subtraction, and point source photometry}

To study GCs, we needed to minimize the contamination due to light from bright galaxies in the fields, i.e., NGC 3115 and NGC 3115-DW01 in one case, and NGC 1399, NGC 1379, NGC 1380, NGC 1381 NGC 1382, NGC 1386, NGC 1387, NGC 1389, NGC 1396, and NGC 1404 for Fornax. To model and subtract the galaxies, we used the ISOPHOTE/ELLIPSE task in IRAF/STSDAS (Jedrzejewski 1987) ${ }^{7}$. After modeling and subtracting the profiles of galaxies, to produce a complete catalog of all sources in the VST field of view, we independently ran SExtractor (Bertin \& Arnouts 1996) on the galaxy-model-subtracted frame for each filter. We obtained aperture magnitudes within a diameter aperture of eight pixels $\left(\sim 11^{\prime \prime} 68\right.$ at OmegaCAM resolution) and applied aperture correction to infinite radius. The aperture correction was derived from the analysis of the curve of growth of bright isolated point-like sources. The photometric catalogs in the three bands were then matched adopting $0{ }^{\prime \prime} .5$ matching radius and are available on the project web-page and on the CDS archive.

\footnotetext{
6 See http://www.na.astro.it/vegas/VEGAS/

VEGAS_Targets.html

IRAF is distributed by the National Optical Astronomy Observatory, which is operated by the Association of Universities for Research in Astronomy (AURA) under cooperative agreement with the National Science Foundation.
}

\subsection{Globular cluster selections}

To select GC candidates we applied photometric, morphometric, and color selection criteria as listed in Table 2, highlighted in Figs. 1-3. In general, the criteria adopted were based on the parameter space occupied by confirmed GCs, UCDs, and stars; the latter were rejected as contaminants from the catalog. As reference for NGC 3115 we adopted the spectroscopic catalog from Arnold et al. (2011) and the photometric catalogs of GCs and UCDs from Jennings et al. (2014). For NGC 1399 we adopted as reference the spectroscopic sample of GCs and stars from Schuberth et al. (2010), and the spectroscopically confirmed UCDs from Mieske et al. (2002, 2004, 2008, and references therein). In the following we explain in more detail the selection criteria adopted.

First, we measured the magnitude concentration index, described in Peng et al. (2011), using the difference in magnitude measured at 6 pixels aperture diameter and at 12 pixels, $\Delta X_{6-12} \equiv \operatorname{mag}_{X, 6 \text { pix }}-\operatorname{mag}_{X, 12 \text { pix }}$, where $X$ is either the $g$ or the $i$ band aperture corrected magnitude. For point-like sources, after applying the aperture correction to the magnitudes at both radii, $\Delta X_{6-12}$ should be statistically consistent with zero.

Figure 1 shows the $g$-band concentration index for the full catalog of ugi matched sources in NGC 3115 (gray dots). Confirmed sources are shown in the left panel with green for GCs, where full/empty circles indicate spectroscopic/photometric confirmed GCs; red for stars; and blue for UCDs. The figure shows that UCDs tend to have $\Delta g_{6-12}>0$ because of their angular size, which is not negligible compared to the PSF size even on VST imaging data ${ }^{8}$.

Then, we compared the morphometric (FWHM, CLASS_STAR, flux radius, Kron radius, Petrosian radius, and elongation) and photometric properties of the full matched ugi catalog with the same properties of spectroscopically or photometrically confirmed GCs, UCDs, and stars available from the literature. Such comparison allows us to identify a membership interval for each class of objects, in particular for GCs, which are more interesting for this study. We adopted a large set of parameters so to exclude anomalous or peculiar sources that might be more efficiently detected with one parameter rather than others, hence allowing us to minimize stellar contamination.

As further photometric selection criteria we rejected sources $\sim 3 \sigma_{\mathrm{GCLF}}$ brighter than the turn-over magnitude, $M^{\mathrm{TOM}}$, and with photometric errors on magnitude and colors higher than a fixed maximum. The bright limit was obtained adopting the $M_{g}^{\mathrm{TOM}}$ and $\sigma_{\mathrm{GCLF}}-M_{z}$ relations from Villegas et al. (2010, their Eq. (9)), assuming $M_{z}=-24$ and $-21.5 \mathrm{mag}$, for NGC 1399 and NGC 3115, corresponding to $(V-z) \sim 0.6$ for both. For NGC 3115 we extended the bright cutoff farther to fainter limits, by 0.5 mag (e.g., from $m_{g} \sim 19.0$ to $m_{g} \sim 19.5$ ), to avoid the expected larger stellar contamination from field stars at the expense of gaining only a handful of bright GCs. Because of the lower absolute Galactic latitude, NGC 3115 is expected to have higher levels of MW stars contamination with respect to NGC 1399 at any given magnitude. We used the Besancon models of the Galaxy (Robin et al. 2003) to obtain an estimate of the stellar contamination as a function of magnitude. The contamination for NGC 3115 is $~ 30 \%$ higher than NGC 1399 at the level of $m_{g} \sim 20 \mathrm{mag}$ and drops to $15 \%$ higher at $m_{g} \sim 25 \mathrm{mag}$. A direct comparison of Figs. 4 and 5 gives visual support to such expectation from models.

\footnotetext{
8 However, see Fig. 3 and Appendix A.1, for some comments on the reliability of the list of UCD candidates in NGC 3115.
} 

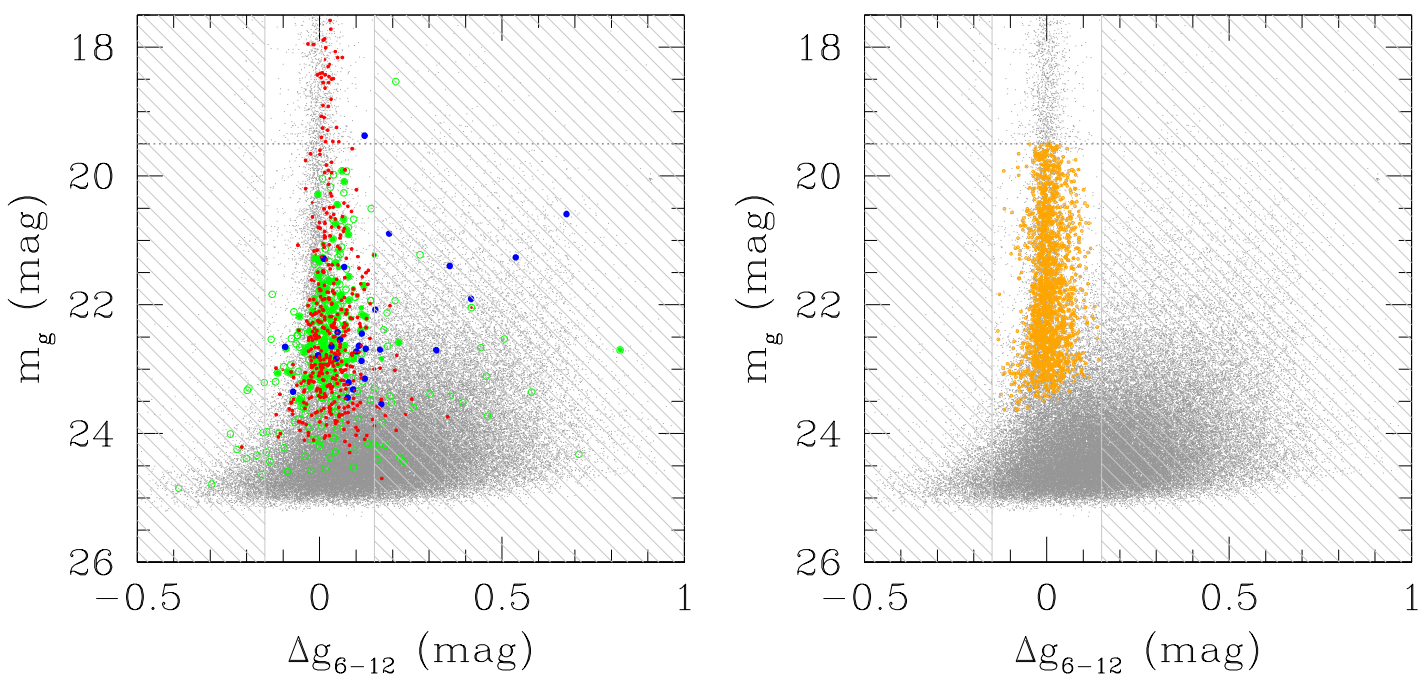

Fig. 1. Left panel: $g$ magnitude vs. concentration index $\left(\Delta g_{6-12} \equiv g_{6 \text { pix }}-g_{12 \text { pix }}\right)$ for the full ugi matched sample (in gray) in NGC 3115. Spectroscopic confirmed GCs are shown with green filled circles (from Arnold et al. 2011) and photometric confirmed GCs from ACS are shown with empty green circles (from Jennings et al. 2014). Blue circles indicate UCDs from Jennings et al. (2014). Red dots indicate confirmed stars from both the spectroscopic and ACS studies cited. The gray hatched area shows the regions of sources rejected based on the concentration index selection. The horizontal dotted line indicates the bright magnitude cut. Right panel: as left panel, except that GC candidates before color-color selection are shown with orange empty circles.
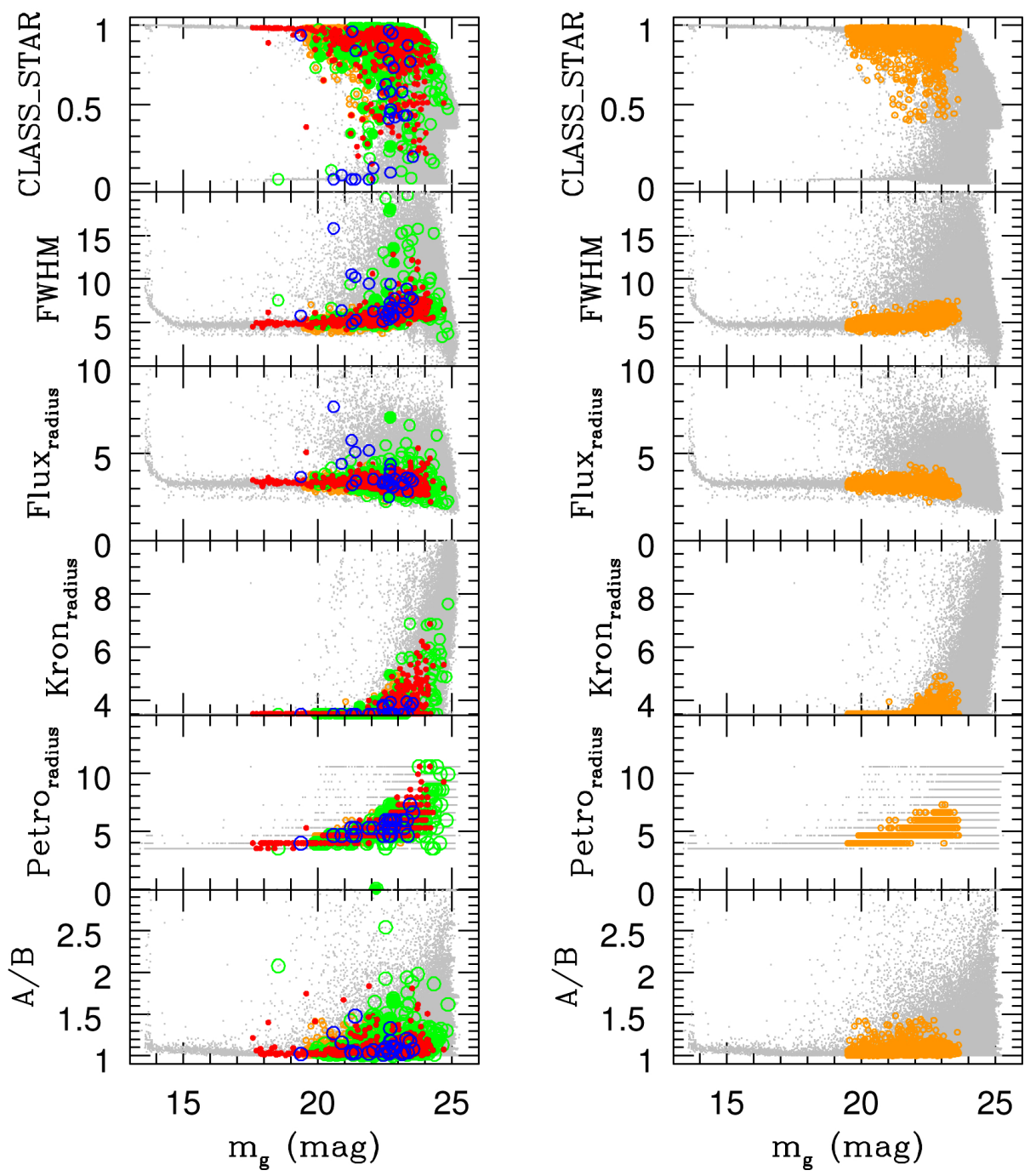

Fig. 2. Left panels: SExtractor output parameters in the $g$-band for the full sample of detected sources in NGC 3115 vs. corrected aperture magnitude (within 8 pixel diameter, gray dots). Color coding is the same as in Fig. 1. Right panels: as left, but selected GC candidates are shown with orange circles. 
Table 2. Photometric and morphometric selection criteria adopted.

\begin{tabular}{lccc}
\hline \hline Quantity & Band & NGC 3115 & NGC 1399 \\
\hline$\Delta X_{6-12}$ & $g / i$ & \pm 0.15 & \pm 0.15 \\
CLASS_STAR & $g$ & $\geq 0.4$ & $\geq 0.4$ \\
CLASS_STAR & $i$ & $\geq 0.7$ & $\geq 0.2$ \\
PSF FWHM & $g$ & $\geq 3.5, \leq 7.5$ & $\geq 4.5, \leq 8.5$ \\
PSF FWHM & $i$ & $\geq 3.5, \leq 7.5$ & $\geq 4.5, \leq 8.5$ \\
Flux radius & $g$ & $\leq 4.5$ & $\leq 4.5$ \\
Flux radius & $i$ & $\leq 4.0$ & $\leq 4.5$ \\
Kron radius & $g / i$ & $\leq 5$ & $\leq 5$ \\
Petro. radius & $i$ & $\geq 3.5, \leq 7.5$ & $\geq 3.5, \leq 7.5$ \\
Elongation a/b & $g / i$ & $\leq 1.5$ & $\leq 1.5$ \\
$(u-i)$ & & $\geq 1.25, \leq 3.75$ & $\geq 1.25, \leq 3.75$ \\
$(g-i)$ & & $\leq 0.4, \leq 1.4$ & $\geq 0.4, \leq 1.4$ \\
$\Delta(u-i)$ & & $\leq 0.3$ & $\leq 0.3$ \\
$\Delta(g-i)$ & $g / i$ & $\leq 0.2$ & $\leq 0.2$ \\
$\Delta$ mag & $g$ & 19.5 & $\leq 0.2$ \\
$m^{\text {bright }}$ & $i$ & 18.5 & 20.2 \\
$m^{\text {bright }}$ & & $|(g-i)-[0.380(u-i)-0.005]| \leq 0.2$ & $|(g-i)-[0.362(u-i)-0.0205]| \leq 0.2$ \\
Color-color & & & \\
\hline
\end{tabular}

Notes. Explanation of listed parameters. $\Delta X_{6-12}$ : interval adopted for the magnitude concentration index in $g$ - and $i$-band. SExtractor output parameters: CLASS_STAR: Neural-Network-based star/galaxy classifier; PSF FWHM: point spread function full width at half maximum; Flux Radius: half light radius; Kron Radius: defined from the automatic aperture photometry, intended to give a precise estimate of total magnitude, inspired to Kron (1980) first moments algorithm; Petro. Radius: the radius at which the surface brightness of the isophote is $\eta$ times the average surface brightness within the isophote; Elongation: semi-major over semi-minor axis ratio (see Bertin \& Arnouts 1996, and references therein for more details). Other selection parameters. $(u-i)$ and $(g-i)$ : colour intervals adopted from the comparison with empirical data and stellar population models; $\Delta(g-i)$ and $\Delta(u-i)$ : maximum allowed error on colors; $\Delta$ mag: maximum error on magnitude; $m^{\text {bright }}:$ bright cut magnitude, at $\sim 3 \times \sigma$ brighter than the turn-over magnitude of the GCLF; color-color: equation of color-color region used for selecting GC candidates.

In the left panels of Fig. 2, we plot the parameters analyzed, together with the selection sample, adopting the same color and symbol coding as in Fig. 1.

The adopted ranges of $\Delta X_{6-12}$ and of the various morphophotometric selection parameters based on the properties of known GCs are given in Table 2. The differences in the selection criteria between the two targets are mostly due to differences in the quality of the imaging data and, also to a lesser extent, to intrinsic differences between the two galaxies, their environment, and their position in the sky ${ }^{9}$.

The right panels in Figs. 1 and 2 show the selected GC candidates with orange circles derived from the morphometric and photometric criteria adopted.

The adoption of the selection described above results in highly efficient identification of point-like sources. As recognizable in the color-color diagram shown in Fig. 3, the catalog of sources obtained using the combination of concentration index and morpho-photometric selections is highly concentrated on the stellar sequence of the color-color diagram (orange dots in the figure) with only little contamination from galaxies ${ }^{10}$. The diagram also shows that the GC sequence overlaps with the sequence of foreground stars in the MW, ranging from $[(u-i),(g-i)]=[\sim 1,0.2]$ occupied by main-sequence turn-off stars, to $[\sim 6,3]$ for low main-sequence stars. To narrow down the stellar contamination, we adopted both the literature GC samples

\footnotetext{
9 No radial cut was imposed to examine the GCs. For NGC 3115 the choice is motivated by the fact that at the galaxy distance the GC system could potentially cover the entire VST frame (see Sect. 3.4). For NGC 1399, instead, the choice is motivated by the fact that also candidate intergalactic GCs are of interest in our analysis (see Sect. 3).

${ }^{10}$ The sequence of galaxies is in upper left in the figures, approximately centered on $(u-i) \sim 1.0$ and $(g-i) \sim 1.0 \mathrm{mag}$.
}

and the simple stellar population models (from the Teramo-SPoT group; Raimondo et al. 2005, in prep.; Raimondo 2009) to identify the region of the $(g-i)-(u-i)$ color-color diagram hosting GCs. The color ranges from both empirical data and stellar population models appear well defined in the color-color plane. We finally adopted slightly wider color intervals to be the most inclusive possible, yet avoiding the increase in the level of contamination toward the blue and red maxima of the GC color-color sequence. The $(g-i)-(u-i)$ region adopted for GC selection is also given in Table 2 for both galaxies.

The analysis of the GCS properties could require the characterization of the completeness functions and the consequent completeness correction to the data. However, the absolute number of GCs is not important for our purposes. It is the relative comparison between the two targets that interest us more. Furthermore, deriving such a correction goes beyond the scope of the present work, both because the complexity of deriving such a function, which would by necessity be color dependent, radial dependent, and also, to some extent, chip-by-chip dependent and because the results presented in the forthcoming sections are not be affected by such correction. The completeness correction would certainly increase the number of GCs, globally and locally, but it would affect the numbers almost homogeneously at all radii, except for the innermost galactocentric radii $\lesssim 1^{\prime}$ (e.g., Kim et al. 2013a), which are mostly populated by red GCs and not of much interest for this work because of the low GC detection rates with VST data at such galaxy radii characterized by high surface brightness levels. Hence, the correction leaves the relative fractions of GCs and the outcome of our analysis essentially unchanged.

The final sample of selected GC candidates, shown with pale blue dots in the right panels of Fig. 3, contains $N_{\mathrm{GC}}=2142$ for NGC 3115 and $N_{\mathrm{GC}}=1792$ for NGC 1399. The full table with 

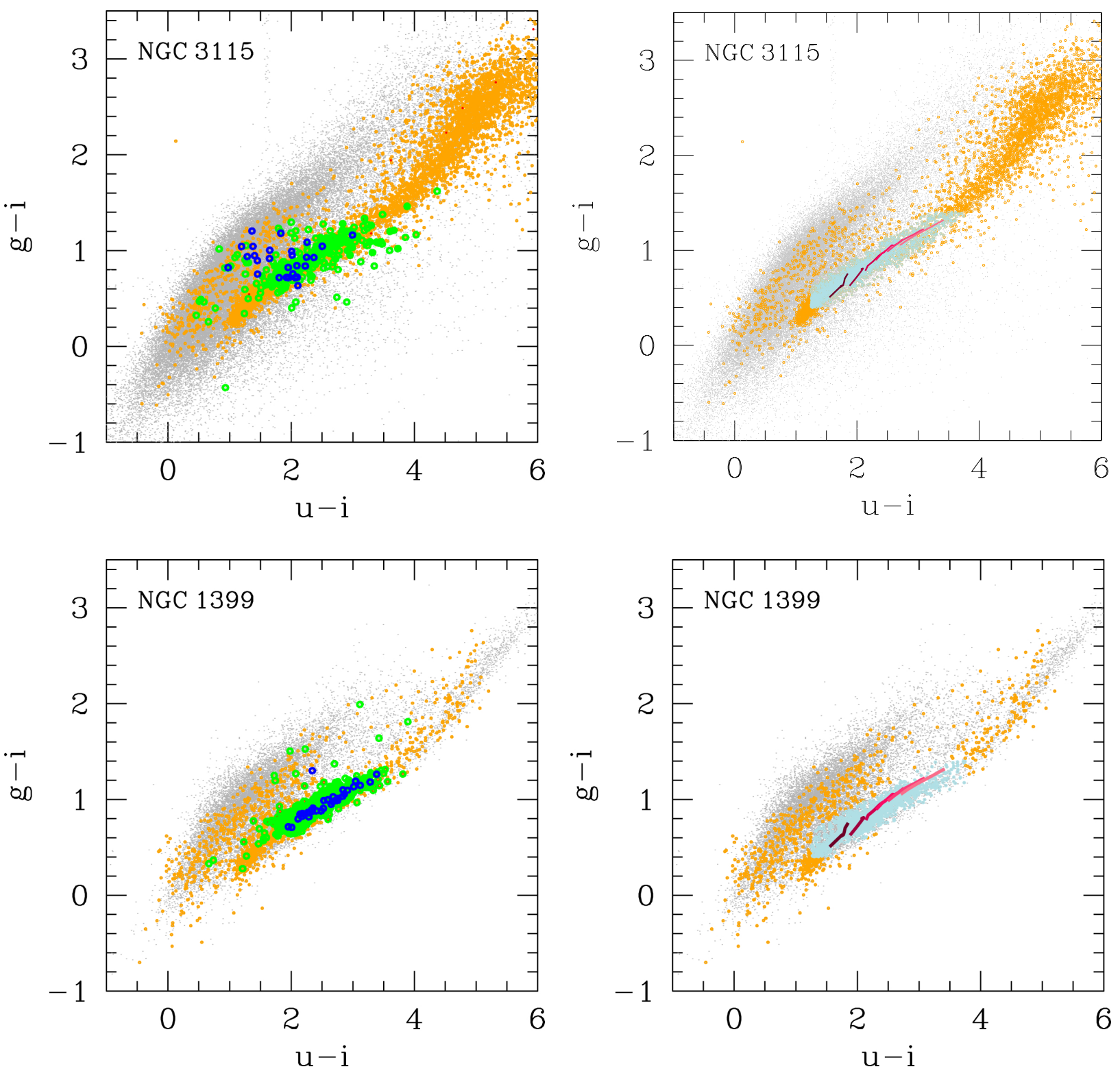

Fig. 3. Upper left panel: color-color diagram for the full catalog of matched sources in NGC 3115 (gray dots) and catalog of GC candidates obtained using the photometric and structural selection criteria shown in Figs. 1-2 (orange circles). Green and blue circles show the GCs and UCDs from the literature, as in previous figures. Even with no color selection, the sequence of orange dots concentrates on the well-defined sequence of compact sources (stars and GCs). Upper right panel: as left, but color selected GC candidates are shown with pale-blue circles. SSP SPoT models for ages between 2 and $14 \mathrm{Gyr}$ and $[\mathrm{Fe} / \mathrm{H}]$ between -2.3 and +0.3 are plotted with solid lines in various purple shades. Lower left: as in upper left panel, but for NGC 1399. In contrast to the UCDs candidates in NGC 3115, the (spectroscopically confirmed) UCDs in Fornax are all well lined up with the color-color sequence of GCs. Lower right: as upper right, but for NGC 1399.

objects coordinates and photometry is available at the CDS and on the VEGAS project pages.

\section{Results}

Using the final sample of matched ugi sources, obtained from the photometric, morphometric, and color selections described in the last Sect. 2.3, we analyzed the two-dimensional spatial distribution, the color distribution, and the azimuthally averaged radial density profiles of GC candidates for the two targets. The color magnitude diagrams for the full matched sample of sources and the selected GC candidates, together with the samples of confirmed GCs, UCDs, and contaminating stars, are shown in Fig. 4 for NGC 3115 and Fig. 5 for NGC 1399.

\subsection{Two-dimensional spatial distribution of GC candidates}

Figure 6 shows the surface density of GC candidates around NGC 3115 (upper, middle, and right panels) and NGC 1399 (lower, middle, and right panels), together with the $g$-band image, shown in the left panels. The GC maps shown in middle and right panels are derived by dividing the surface area in equally spaced RA and Dec bins, and then counting the number of GC candidates per unit area. We adopted a binning size of $21^{\prime \prime} \times 21^{\prime \prime}(100 \times 100$ pixels $)$ for the middle panels and $52.5^{\prime \prime} \times 52.5^{\prime \prime}(250 \times 250$ pixels $)$ for the left panels of the figure $^{11}$. The resulting bidimensional maps were then smoothed

${ }^{11}$ At the adopted distances, 1" corresponds to $\sim 45$ pc for NGC 3115 and to $\sim 97$ pc for NGC 1399 . 

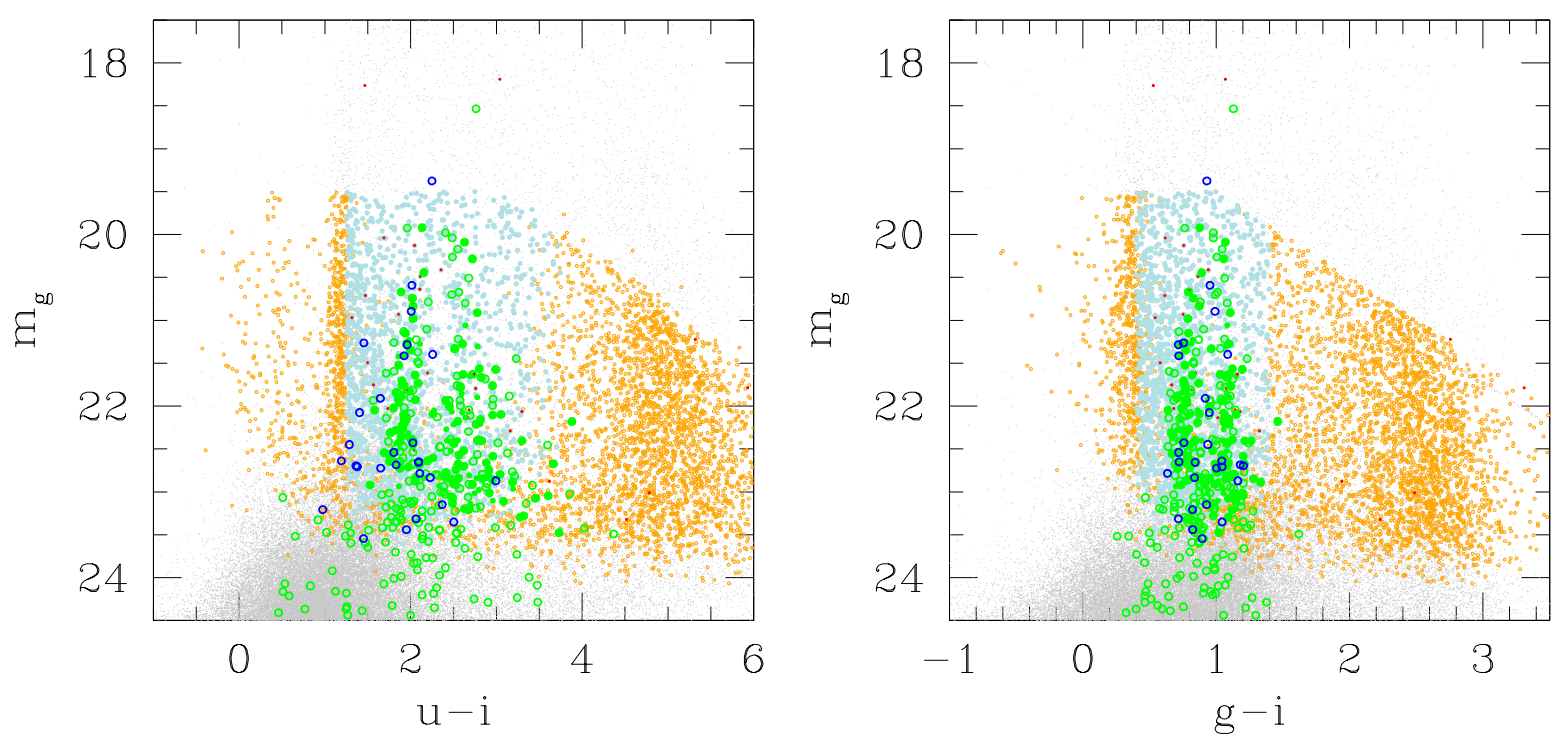

Fig. 4. Color-magnitude diagram for the sources in the field of NGC 3115. Symbols are the same as in Fig. 3: gray dots refer to the full matched sample, orange empty circles to sources selected using the range of morphometric and photometric properties of confirmed GCs, pale-blue filled circles are the sources in the final catalog. Confirmed sources, taken from the literature, are shown with green circles (GCs), red dots (stars), and blue empty circles (UCDs).
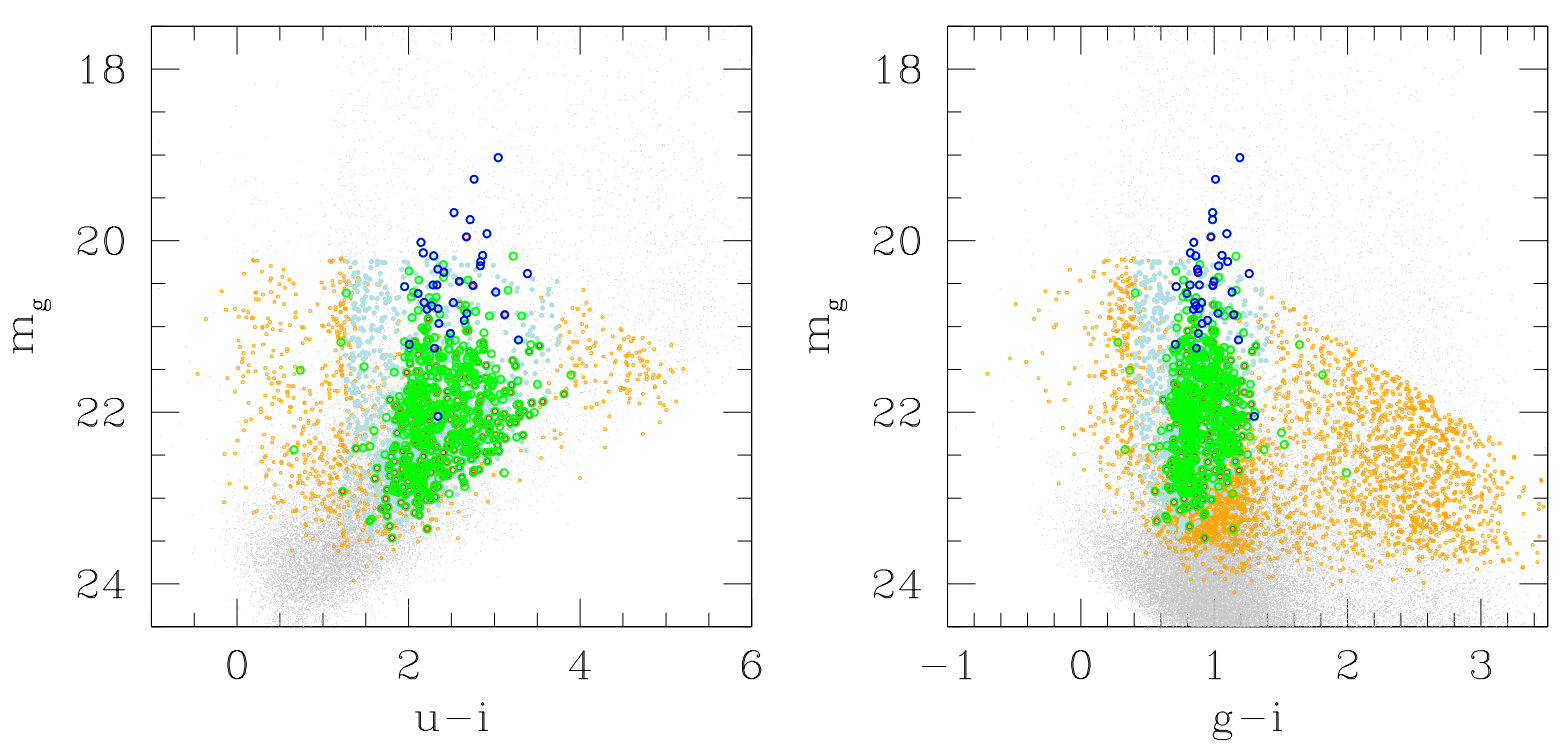

Fig. 5. As in Fig. 4, but for NGC 1399.

with Gaussian kernels adopting a standard deviation $\sigma=2$ in units of grid spacing.

For NGC 3115, the first panel also shows the isodensity contour levels obtained from the $21^{\prime \prime} \times 21^{\prime \prime}$ smoothed GC density map. The surface density maps for this galaxy show several noteworthy features. The presence of nonzero background (zero counts correspond to violet color in the panels, highest GC count rates to red), at even very large galactocentric radii, testifies the level of contamination affecting the sample mainly caused by foreground MW stars. Even over the large area considered here, such stellar contamination is either constant or changes very smoothly with respect to the local density of GCs. Therefore, the GC system of NGC 3115 and of its dwarf companion, which is NGC 3115-DW01 southeast of NGC 3115, clearly emerges over the contaminating sources. An overdensity of GCs also appears in the region of the dwarf spheroidal KK 084, which is a dwarf galaxy with high specific frequency of GCs $\left(S_{N} \sim 10\right.$; Puzia \& Sharina 2008, white cross $\sim 5.5^{\prime}$ east of NGC 3115 ). The GC overdensity is better seen in the upper middle panel of Fig. 6 .

Two relevant points for the light and GC maps of NGC 3115 seen in Fig. 6 are the similarity in terms of elongation and inclination between the GC density profile and light profile of field stars, and the larger spatial extent of GCs compared to galaxy light. While the latter is a long-known observational property of GCSs, the common GCs and galaxy light geometry is more surprising, as the system of halo GCs, at large galactocentric distances, is usually pictured as spherical. Nevertheless, several studies have found azimuthal distributions of GCs mirroring the galaxy (Kissler-Patig et al. 1996; Park \& Lee 2013; Wang et al. 2013; Hargis \& Rhode 2014; Kartha et al. 2016). At the level of surface brightness depth of our data $\left(\mu_{g} \sim 29.5 \mathrm{mag} \mathrm{arcsec}{ }^{-2}\right.$; Spavone et al., in prep.), we do not observe any obvious bridge 

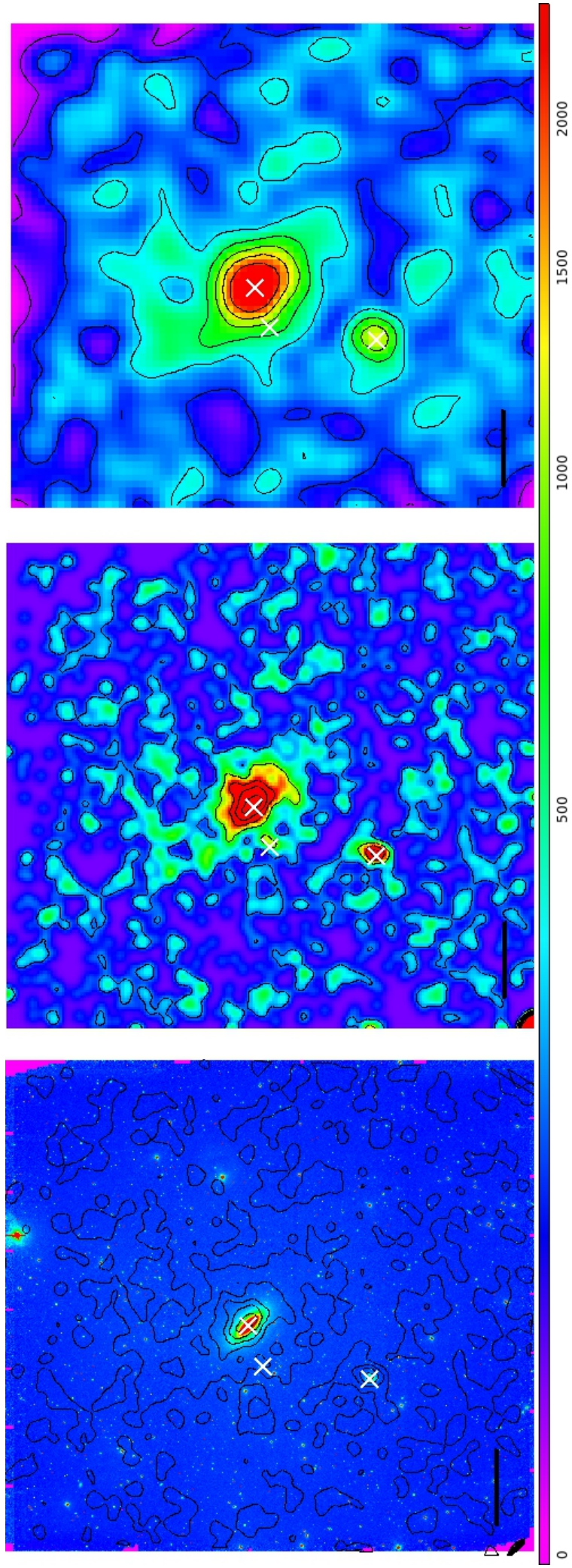

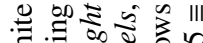

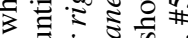

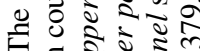

당 इ

于े 0.

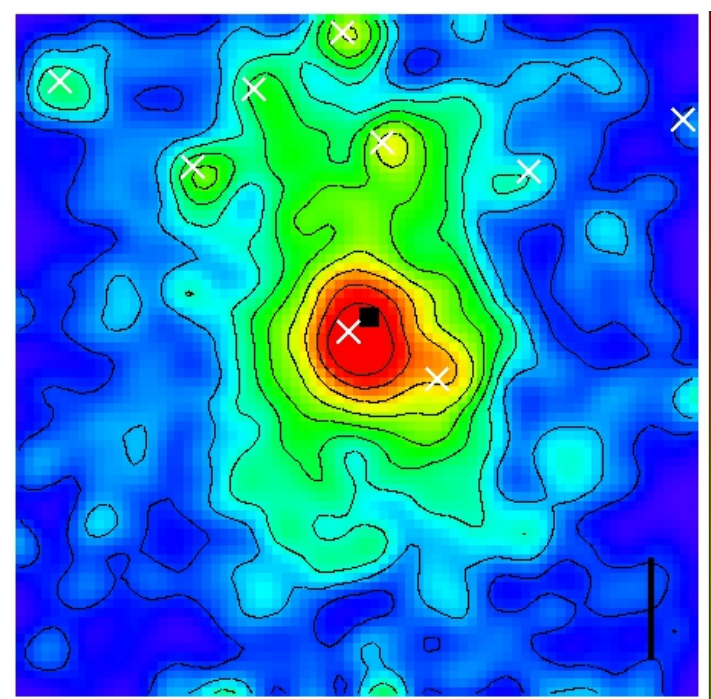

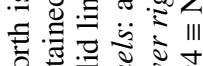

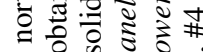

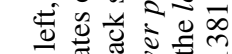

을

$=$ 의

出

论

낭

政

열

药

도을

政

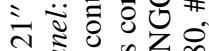

$x=20$.

त

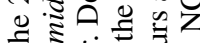

घ डิ

원혀웡

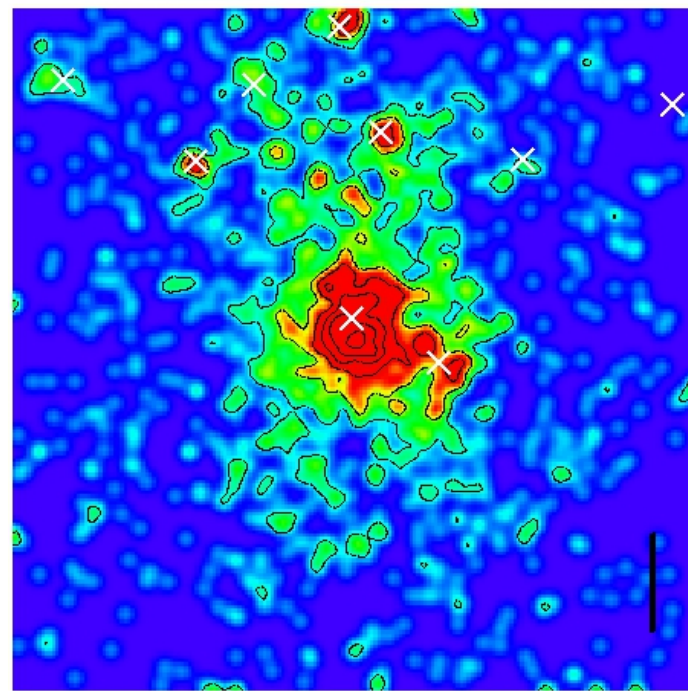

구을

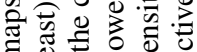

$\sin 30$

?

可 2

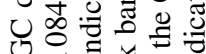

o

政

ह

的政

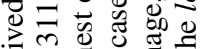

Do

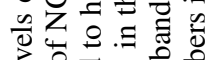

은항

政记

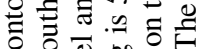

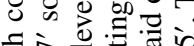

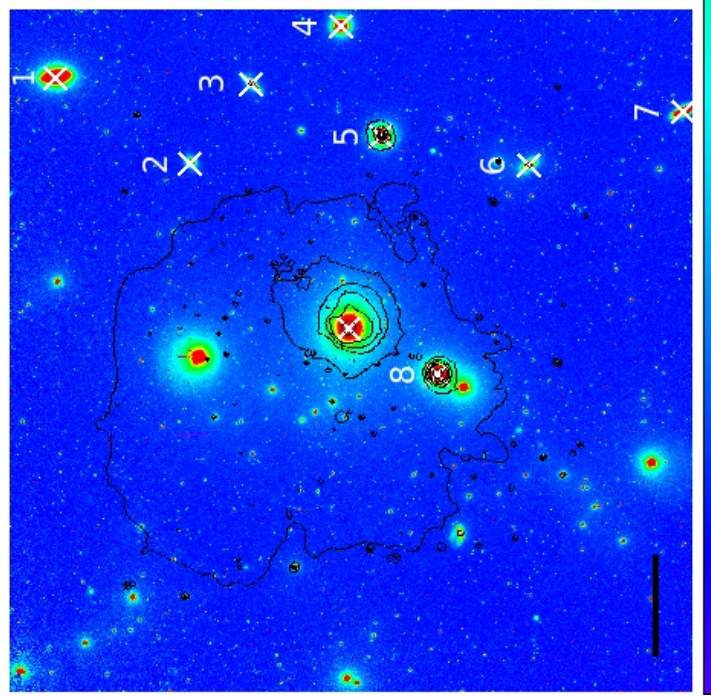

言交

원

के

ว

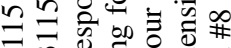

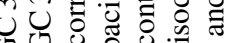

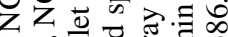

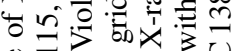
品 品 乙的焉 흔 政记 o. 0.00 $\because: 0.00$ छ윰 5. ङ

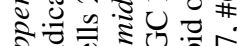
s.

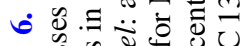

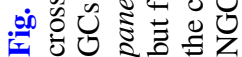


of diffuse light between NGC 3115 and NGC 3115-DW01, nor do we find any obvious enhancement of GC counts between the two galaxies, even though the density contours of GCs around the dwarf companion appear elongated in the direction of NGC 3115.

The surface density plots in the lower panels of the figure confirm the results from previous studies on NGC 1399, revealing the presence of substructures in the spatial distribution of the GC population, such as the overdensity bridging the GCS in NGC 1399 with NGC 1387, or NGC 1381, or the southeastern arc (feature G in D'Abrusco et al. 2016) ${ }^{12}$.

As observed in the lower middle and left panels of Fig. 6, in addition to local density maxima associated with the bright galaxies in the field (mainly around NGC 1379, NGC 1380, NGC 1381, NGC 1382, and NGC 1387, besides NGC 1399), the spatial extent of the GC overdensity covers a large portion of inspected area, providing supporting evidence in favor of its intergalactic origin. The morphology of the overdensity is asymmetric with an elongated east-west shape. For the regions west of NGC 1399, Iodice et al. (2017) observed the presence of an overdensity in the galaxy light profile as well, and suggested the presence of a stellar stream between the galaxy and the close bright neighbor NGC 1387. We confirm the presence of the substructures, some already presented and discussed in Bassino et al. (2006) and D'Abrusco et al. (2016). To model the GC density profile, we ran ELLIPSE on the density maps and found that the centroid of the distribution within the isodensity radius $\sim 25^{\prime}$ is $\sim 3^{\prime}$ southwest offset with respect to the photometric center of NGC 1399 (black square in the lower right panel in Fig. 6), with position angle PA $\sim-85 \mathrm{deg}$ and ellipticity $\epsilon \sim 0.4$. At smaller galactocentric radii, within $\sim 5^{\prime}$, the offset is $\lesssim 1^{\prime}$ in the opposite direction, east of NGC 1399 centroid, consistent with the results from Kim et al. (2013a) with PA $\sim-70$ deg and $\epsilon \sim 0.15$. A similar GC distribution offset has also been recently found in Coma by Cho et al. (2016). We also observe that centroid of NGC 1399 GC distribution does not coincide with the maximum value of the GC distribution (close to NGC 1399 center), mostly because the asymmetric east to west distribution of GCs is overdense on the west side of the cluster, where most of the bright companions of NGC 1399 are located.

Analyzing ROSAT X-ray data, Paolillo et al. (2002) highlighted the presence of three distinct components in the core of Fornax: a central cooling flow region, the galactic halo, and the cluster-wide X-ray halo. In the first lower panel of Fig. 6 we overlaid the $g$-band VST image of NGC 1399 with the X-ray contours, derived from Scharf et al. (2005), showing the softband (0.3-1.5 keV) mosaic obtained with Chandra/ACIS. On the scale of $z 30^{\prime}$, the southwest to northeast elongation of $\mathrm{X}$-ray contours first observed from ROSAT is also confirmed by the data from Chandra and provides supporting evidence for a northeastern displacement of the hot gas in the cluster with respect to the optical properties of galaxies (D'Abrusco et al. 2016; Iodice et al. 2016). Such geometry brought up the idea that the Fornax core, and the subcluster hosting NGC 1316, may lie along a filamentary structure that is flowing in toward a common barycenter (Drinkwater et al. 2001; Scharf et al. 2005). The lack of immediate correspondence between the X-ray southwest to

\footnotetext{
12 In contrast to our previous analysis, presented in D'Abrusco et al. (2016), where we focused on large-scale ( degrees) substructures in the spatial distribution of bright GCs selected using PCA analysis, here we i) apply different selection criteria for GCs; ii) are more interested in the bulk appearance of the GCS on a smaller area than in D'Abrusco et al.; and iii) revisit the analysis with specific focus on the comparison between the GCSs in NGC 1399 and NGC 3115.
}

northeast elongation and the east to west elongation of the intracluster GCs is not surprising; given the collisional/noncollisional nature of gas/galaxies, they react differently to the possible ongoing interaction with the Fornax A subgroup.

On smaller angular scales $\left(\lesssim 10^{\prime}\right)$ X-ray and GCs contour maps for NGC 1399 appear more similar, as both components appear approximately elongated in the southeast to northwest direction (lower left and middle panels in Fig. 6), nearly orthogonal to that on a larger scale, connecting NGC 1404 to NGC 1399 and beyond. The large-to-small scale asymmetry of the gas distribution could be due to the motion of NGC 1399 in the diffuse cluster halo, while for GCs the differences between large- and small-scale distributions might reflect the difference between intracluster GCs and the complex of interactions of the GCS in NGC 1399 with the GCS in NGC 1404 and other close galaxies (Bekki et al. 2003).

\subsection{Spatial distribution of blue and red GC candidates}

For both fields we also separately analyzed the surface density maps of blue and red GCs, adopting the dividing color at $(u-$ $i)=2.3$ for NGC 3115 GCs and $(u-i)=2.5$ for NGC 1399 based on the average dip between the Gaussian best matching the distribution of the two subpopulations over the entire field of view (see next Sect. 3.3). The maps are shown in Fig. 7 (upper panels for NGC 3115, lower panels for NGC 1399).

The shape of blue and red GC maps for NGC 3115 do not differ much from each other, except for the larger angular extent of the blue subcomponent, which is a well-known feature in individual galaxies (Geisler et al. 1996; Côté et al. 2001; Cantiello et al. 2007). The surface distribution for red and blue GCs appears approximately concentric to galaxy light and aligned with the major axis of the galaxy. This is illustrated in the upper panels of Fig. 7, showing the density maps of blue (left panel) and red (middle panel) GC candidates, adopting the $52.5^{\prime \prime}$ binning grid.

The blue and red GC maps for NGC 1399 are notably different form each other. We find that the red component is mostly concentrated around the bright galaxies closest to NGC 1399 (white crosses in the figure), and the blue GC component appears more extended, still showing the densest peaks close to the positions of bright galaxies.

As further evidence for the intergalactic nature of the blue GC component, we note that west of NGC 1399 there are several bright galaxies within a radius of $\sim 30^{\prime}$, thus the enhancement of blue GCs might come from the overlap of the outer envelopes of blue GCs host by the single galaxies in the region. However, for the GC enhancement toward east, the overabundance of blue GCs cannot be originated by the overlap of galaxy-hosted GCs because of the lack of bright galaxies in the area.

Previous studies discovered a large population of GCs in galaxy clusters, which appears not to be associated with individual galaxies, numerically dominated by blue GCs, in relative fraction 4:1 (Peng et al. 2010). To further verify whether the blue GC overdensity in NGC 1399 (left panel in Fig. 7) is due to the overlap between the intrinsically more extended blue GC distribution of individual galaxies with respect to the red GC distribution, or if is truly an extra intergalactic component, we made an attempt to generate a blue-to-red GC residual map. First, we rescaled the red GC density map to match the blue GC density of NGC 1399 at peak and at galactocentric radius $r_{\text {gal }}=7^{\prime}$. Then, by subtracting the rescaled red GC map to the blue map, we obtained the residual map shown in lower 


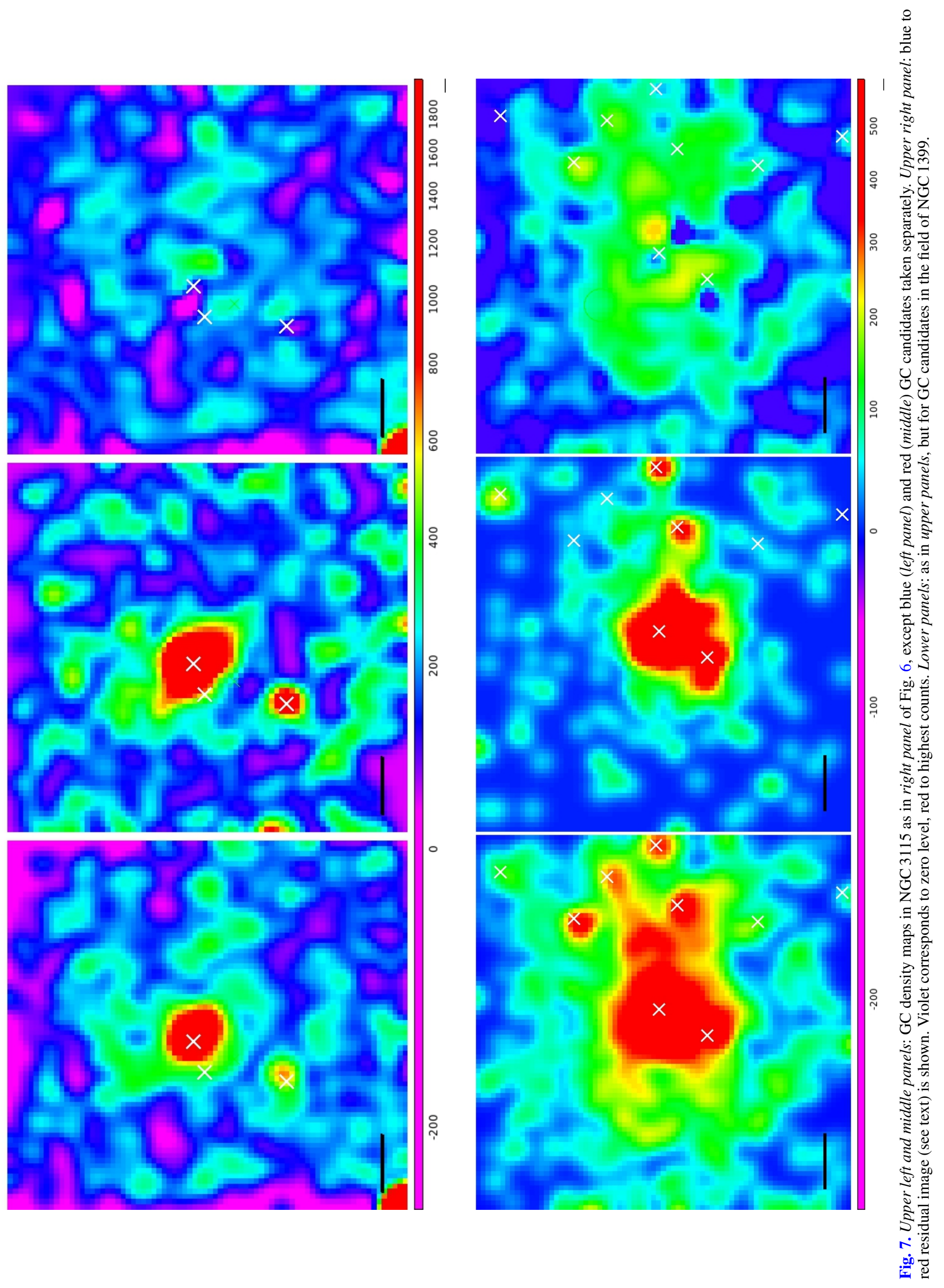


right panel of Fig. 7. With this qualitative approach, we expected any overdensity of blue or red GCs to appear in the residual image. To verify such expectation, the same procedure was applied to NGC 3115 (upper right panel of the figure) because for this galaxy the GCS is basically featureless. Indeed, the residuals do not show the elongated structure associated with the GC overdensities present in the first two panels of the figure. The GC overdensity close to NGC 3115-DW01 is reduced too, although the red-to-blue scaling is properly derived only for the GCs in NGC 3115. Within the limits of the approach adopted, the GC residual density map for NGC 1399 clearly shows an overdensity stretching along the east-west side of the cluster. Compared with the X-ray maps discussed above, on cluster-wide scale blue GCs appear to cover the region of the diffuse X-ray emission from hot gas, extending on the east side of the Fornax galaxy cluster, where no bright galaxy with a rich GCS exist. Following the results on the similarity between the X-ray and blue GC profiles in NGC 1399 (Hilker et al. 1999; Forte et al. 2005), Forbes et al. (2012) recognized a general coherence of blue GC density distributions with the X-ray profiles of bright early-type galaxies. Blue GCs stretch on the east-west direction with a relatively symmetric distribution around the cluster core, while the hot gas from X-rays shows a northeast to southwest asymmetric drift with respect to NGC 1399. We speculate that the emerging pattern is that the two Fornax subclusters are falling toward each other with the galaxies and halo GCSs moving ahead of the gas component because of their already mentioned noncollisional nature.

\subsection{Color bimodality and color-color relations}

As mentioned above, NGC 1399 is one of the first galaxies suggested to show evidence of color bimodality in its GCS and is among the most thoroughly studied targets on this specific research topic (Ostrov et al. 1993, 1998; Kissler-Patig et al. 1997, 1999; Dirsch et al. 2003; Bassino et al. 2006). For this galaxy, recent observational studies have shown the lack of coherence of the color distribution with different color indices (Blakeslee et al. 2012; Kim et al. 2013a), favoring the role of nonlinear color-metallicity relations in shaping the observed color distributions, against the classical scenario of a bimodal metallicity distribution generating the observed CB.

NGC 3115, in contrast, is the first external galaxy with $\mathrm{CB}$ for which all observational evidence converges unambiguously toward the existence of a bimodal metallicity distribution of the GCS (Brodie et al. 2012; Cantiello et al. 2014).

In Fig. 8 we plot the $(u-i)$ and $(g-i)$ color distributions for NGC 3115 and NGC 1399, obtained by inspecting various annular regions with the same geometry of the galaxy isophotes (taken from Arnold et al. 2011; Spavone et al., in prep. for NGC 3115; from Iodice et al. 2016 for NGC 1399).

The first column of the panels shows the position of GC candidates in NGC 3115 starting from $r\left({ }^{\prime}\right)=0$ (lowermost panel) out to $25^{\prime}$ (uppermost panel), with $5^{\prime}$ steps. The GC candidates in each annulus are plotted as filled black circles. The lowermost panel also shows the position of the full sample of GC candidates at galactocentric distance larger/smaller than $r_{\mathrm{bg}}=29^{\prime}$ with black/gray dots. The second and third columns of the panels in the figure show surface density histograms vs. $(u-i)$ and $(g-i)$ color, corrected for background density using the candidates at $r \geq r_{\text {bg }}: \Sigma_{\text {corr }}=\left[\Sigma\left(r_{\text {out }}\right)-\Sigma\left(r_{\text {in }}\right)\right]-\Sigma\left(r>r_{\text {bg }}\right)$, where $\Sigma(r)=N_{\mathrm{GC} \text { cand. }}(r) / \operatorname{Area}(r)$ is given in units of counts per square arcminute. For each color the background density distribution is shown with gray shaded histogram in the lowermost panels. The density distributions are corrected for the masked area of the GCs close to the companion galaxy NGC 3115-DW01.

Each row of the panels refers to a different annulus, as shown in the spatial distribution panel (left) and also labeled in the right panels. For sake of clarity, the density histograms with maximum density $\Sigma_{\text {corr }}<0.2$ are multiplied by a factor of 10 and are shown with dotted lines ${ }^{13}$.

The diagram for the innermost annulus of NGC 3115 shows the unambiguously bimodal color distribution, especially for the $(u-i)$ color that reveals a marked dip at $(u-i) \sim 2.3$ mag. At increasing radii, the distribution shows the behavior already discussed in Cantiello et al. (2015): the blue peak moves to bluer colors and the density of red GC decreases with respect to blue GCs. In the last annulus shown, $20 \leq r\left({ }^{\prime}\right) \leq 25$, there is only a residual of candidates with $(u-i) \sim 2.0$ mag and the corrected density is $\Sigma_{\text {corr }} \sim 0.022$ GCs per square arcmin, which can be compared with the statistical error $\delta \Sigma_{\text {corr }} \sim$ $0.011 \mathrm{GCs} \mathrm{sq}^{-1}$. arcmin; this error is estimated assuming Poisson statistic for GC counts and assuming 5\% uncertainty on the estimates of the area for both the annular region and the background.

The rightmost three columns of Fig. 8 show the same analysis for NGC 1399. Also in this case we rejected all sources close to the bright galaxies in the field (indicated with gray crosses in the left panels of the figure). For the correction of background contamination, we adopted $r_{\mathrm{bg}}=32^{\prime}$, although the distributions do not change substantially for larger background radii. Given the nearly twice larger distance of NGC 1399 compared to NGC 3115, to preserve the linear size of the area inspected for the comparison, the angular size adopted for the annuli is half that used for NGC 3115.

The first obvious feature that emerges for the $(u-i)$ and $(g-i)$ color distributions of NGC 1399 (last two columns in Fig. 8) is the less marked gap between the distributions of the blue and red GC peaks.

To quantify the differences between the two GCSs in terms of the color distributions, we used the Gaussian mixture modeling code (GMM; Muratov \& Gnedin 2010) ${ }^{14}$. The analysis is limited to the two innermost annuli, where the relative fraction of background contamination is much more negligible than at larger galactocentric radii.

Table 3 presents the results of the GMM analysis, which is accompanied by the results of the dip test ${ }^{15}$. For seven of eight inspected GC samples, negative kurtosis, large peak separations (in units of the fitted $\sigma \mathrm{s}$ for the two Gaussian), and low $p\left(\chi^{2}\right)$ values from the GMM tests confirm the preference for

13 The residuals for some histogram bins are $<0$, as is easily seen in the dotted histograms. Such negative counts are consistent with zero within estimated uncertainties.

${ }^{14}$ GMM uses the likelihood-ratio test to compare the goodness of fit for double Gaussian vs. a single Gaussian. For the best-fit double model, it estimates the means and widths of the two components, their separation DD in terms of combined widths, and the kurtosis of the overall distribution. In addition, the GMM analysis provides the positions, relative widths, and fraction of objects associated with each peak. It also provides uncertainties based on bootstrap resampling. Blakeslee et al. (2012, see their Sect. 4.2) provide a discussion on the issues inherent to bimodality tests.

15 The dip statistic is a further test of unimodality, proposed by Hartigan \& Hartigan (1985), based on the cumulative distribution of the input sample, and its maximum distance with a best-fitting unimodal distribution. The test searches for a flat step in the cumulative distribution of the input function, which corresponds to a dip in the histogram representation. 

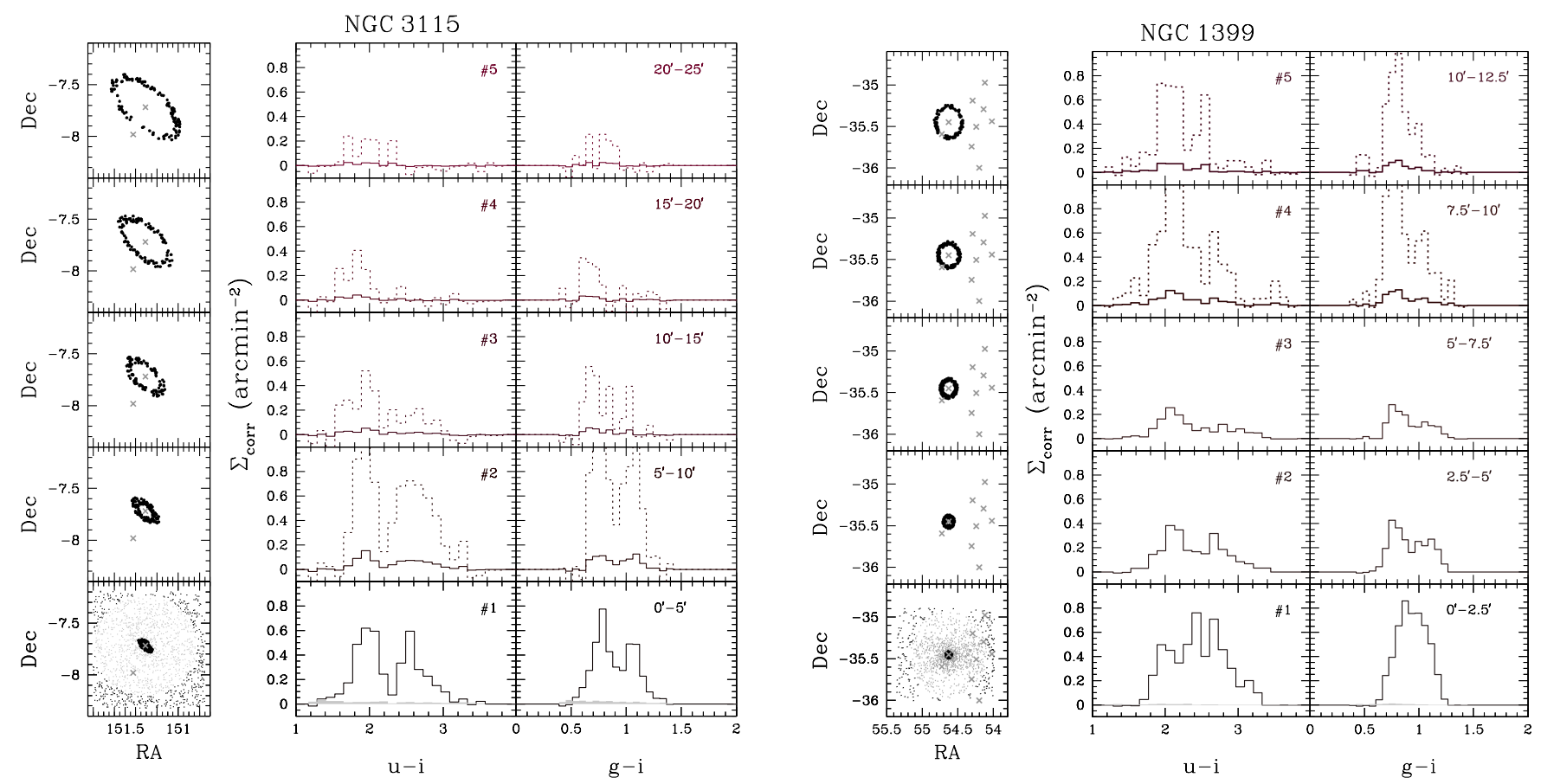

Fig. 8. First column of panels from the left: position of GC candidates selected for color analysis. Each panel shows a different annular region, adopting the geometry of galaxy isophotes. In the lower panel we plot the full sample of selected GC candidates, adopting gray/black color for object within/outside the adopted background radius $r_{\mathrm{bg}}=29^{\prime}$. The gray crosses also indicate the centroid of NGC 3115 and its companion dwarf NGC 3115-DW01. Second column of panels: $(u-i)$ surface density distribution of GCs, corrected for background, at different galactocentric radii (as shown in left panel, and labeled in right panels). Dotted lines show the density histograms with maximum density $\Sigma_{\text {corr }}<0.2$ multiplied by a factor of 10. The gray histogram in the lowermost plot refers to background sources. Third column of panels: as second, but for $(g-i)$ color. Fourth to last column: as first three columns of panels, but for the GC candidates in the field around NGC 1399. The background radius adopted is $r_{\text {bg }}=32^{\prime}$.

Table 3. GMM and Dip test results.

\begin{tabular}{ccccccccccc}
\hline \hline $\begin{array}{c}\text { Target } \\
(1)\end{array}$ & $\begin{array}{c}\text { Color } \\
(2)\end{array}$ & $\begin{array}{c}\text { Ann. } \\
(3)\end{array}$ & $\begin{array}{c}N_{\mathrm{GC}} \\
(4)\end{array}$ & $\begin{array}{c}\text { Blue } \\
(5)\end{array}$ & $\begin{array}{c}\text { Red } \\
(6)\end{array}$ & $\begin{array}{c}f_{\text {red }} \\
(7)\end{array}$ & $\begin{array}{c}\text { Kurt } \\
(8)\end{array}$ & $\begin{array}{c}\text { DD } \\
(9)\end{array}$ & $\begin{array}{c}p\left(\chi^{2}\right) \\
(10)\end{array}$ & $\begin{array}{c}p(\text { Dip }) \\
(11)\end{array}$ \\
\hline NGC 3115 & $(g-i)$ & 1 & 166 & $0.765 \pm 0.015$ & $1.064 \pm 0.022$ & 0.417 & -0.723 & $3.02 \pm 0.43$ & 0.001 & 0.78 \\
NGC 3115 & $(g-i)$ & 2 & 116 & $0.743 \pm 0.025$ & $1.079 \pm 0.021$ & 0.415 & -0.747 & $3.01 \pm 0.45$ & 0.010 & 0.95 \\
\hline NGC 1399 & $(g-i)$ & 1 & 103 & $0.834 \pm 0.056$ & $1.036 \pm 0.059$ & 0.492 & -0.818 & $2.38 \pm 0.34$ & 0.275 & 0.02 \\
NGC 1399 & $(g-i)$ & 2 & 149 & $0.777 \pm 0.012$ & $1.059 \pm 0.020$ & 0.465 & -1.149 & $3.48 \pm 0.35$ & 0.001 & 0.81 \\
\hline NGC 3115 & $(u-i)$ & 1 & 166 & $1.909 \pm 0.029$ & $2.619 \pm 0.110$ & 0.472 & -0.439 & $2.81 \pm 0.75$ & 0.001 & 1.00 \\
NGC 3115 & $(u-i)$ & 2 & 116 & $1.883 \pm 0.050$ & $2.698 \pm 0.072$ & 0.463 & -0.812 & $2.94 \pm 0.36$ & 0.014 & 0.83 \\
\hline NGC 1399 & $(u-i)$ & 1 & 103 & $1.992 \pm 0.112$ & $2.570 \pm 0.142$ & 0.797 & -0.772 & $2.34 \pm 0.34$ & 0.077 & 0.50 \\
NGC 1399 & $(u-i)$ & 2 & 149 & $2.079 \pm 0.038$ & $2.738 \pm 0.065$ & 0.475 & -0.772 & $2.82 \pm 0.40$ & 0.001 & 0.81 \\
\hline
\end{tabular}

Notes. Columns list: (1-3) Target, color and annulus analyzed; (4) number of GC candidates in the annulus; (5) mean and uncertainty of the first, blue, peak; (6) mean and uncertainty of the second, red, peak; (7) fraction of the GC candidates GMM associated to the second peak; (8-9) kurtosis, Col. (8), and separation, Col. (9), between the peaks in units of the fitted $\sigma$ parameters. Values of DD larger than $\sim 2$, and negative kurtosis are necessary but not sufficient conditions for bimodality; (10) GMM value indicating the significance of the preference for a double Gaussian distribution over a single Gaussian (lower $p\left(\chi^{2}\right)$ are more significant; $p\left(\chi^{2}\right)$ close to unity means unimodal distribution is preferred over bimodal); (11) significance level with which a unimodal distribution can be rejected based on the Dip test (high $p$ (Dip) are more significant).

a bimodal Gaussian model over a unimodal distribution for both galaxies. As also revealed by visual inspection, the first annulus of NGC 1399 shows that color distributions are different in $(g-i)$ and $(u-i)$. For the $(g-i)$ color, the distribution visually appears to be unimodal and, despite negative kurtosis and the relative separation of the fitted peak positions DD $>2, p\left(\chi^{2}\right) \sim 0.27$ indicates a marginal preference for the unimodal over the bimodal distribution. For the $(u-i)$, the GMM tests on the selected GCs sample show that the distribution is best represented by a multimodal function with three peaks at $\sim 2.0, \sim 2.4, \sim 2.8$, hosting a fraction of $\sim 31 \%, \sim 27 \%, \sim 42 \%$ of the GC population. For a further check, we adopted the catalog of GC candidates from Kim et al. (2013b), selecting only sources within $\sim 2.5^{\prime}$ from NGC 1399 center; we found that for such sample a distribution with three Gaussian with peaks at $U-I \sim 1.6,1.9,2.5 \mathrm{mag}$, with $26 \%, 27 \%$, and $47 \%$ of the GC population, also provides a good representation of the observed color distribution, although it is not preferred over the bimodal distribution. The complex structure of color distributions for GCs in the innermost regions of NGC $1399\left(r<2.5^{\prime}\right)$ was also discussed by Blakeslee et al. (2012), in a study based on HST ACS and WFC3 data. The authors recognized differing bimodalities in different colors, including the preference for unimodal color distribution in selected GCs subsamples and for given color selections. The two colors 

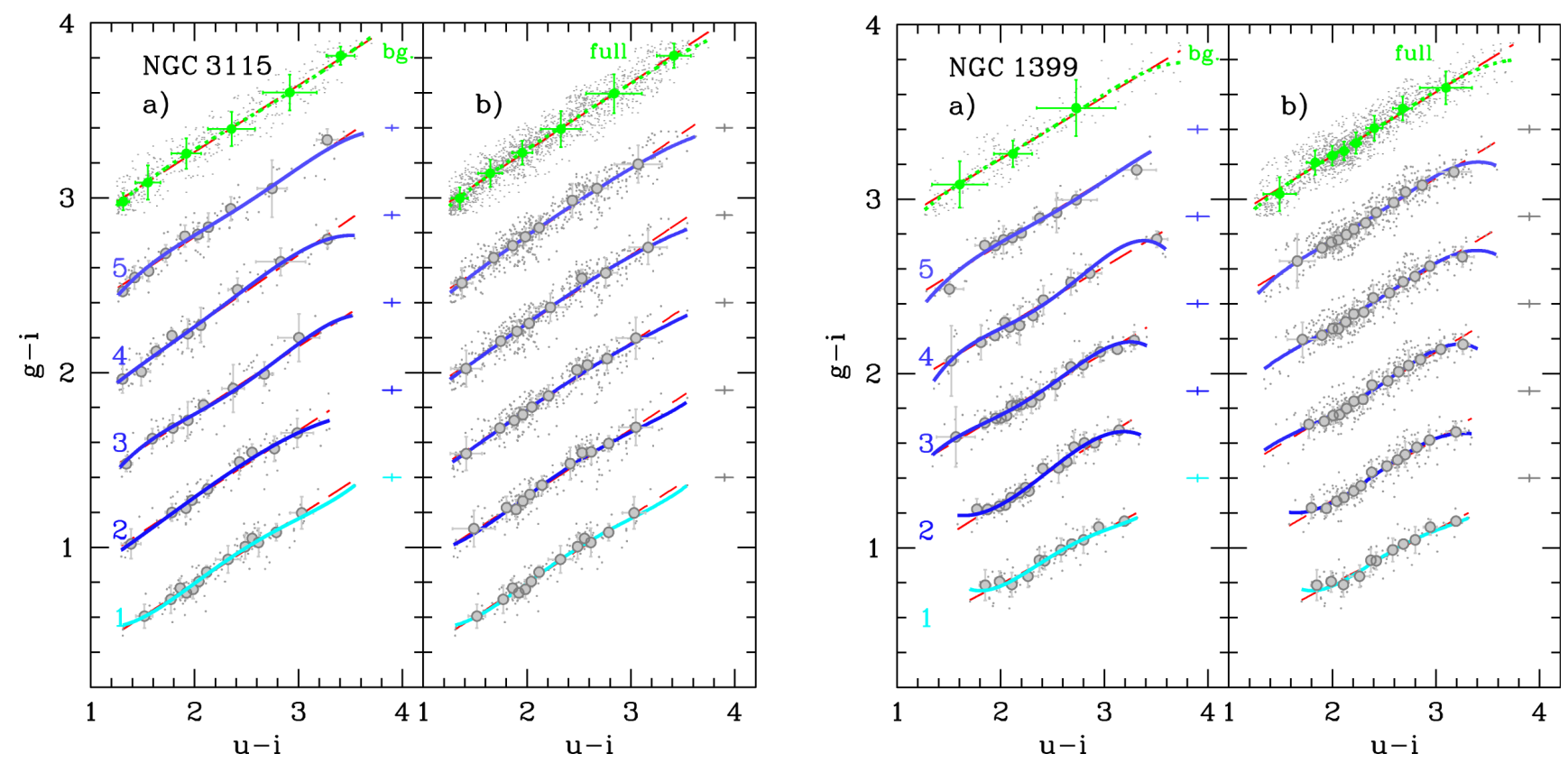

Fig. 9. Left. Panel a: color-color relations fits for the GC candidates in the five annular regions analyzed in NGC 3115 . The CCR for the innermost annulus is the lowermost. Single GC candidates are shown with dots; filled gray circles mark the running median, with rms error bars derived using the maximum absolute deviation. The $(g-i)$ of other annuli are shifted by multiples of +0.5 mag at increasing radii. Annuli are also labeled in the figure. Linear fits are shown with red dashed lines, polynomial fits with solid curves of different shades of blue. The uppermost fits show the CCRs of background sources (red dashed and green-dotted line for linear and polynomial fits; $b g$ label). The median color error for the sources used to derive the CCR fits is reported on the right of each fit. Panel $b$ : as in panel $a$, except that the cumulative sample of candidates is taken within each radius (see text). The uppermost curves are obtained from the full sample of candidates. Right: as in left panels, but for NGC 1399.

inspected here, $(u-i)$ and $(g-i)$, have different relative sensitivity to metallicity changes (more pronounced for $(u-i)$ ), and the lack of correspondence between different color distributions of the same GC population was one of the motivations that led to the projection scenario aforementioned (e.g., Cantiello \& Blakeslee 2007).

Furthermore, by comparing of the dip statistics for the same annuli and colors of both targets, the test highlights the presence of a more obvious step in the cumulative distribution function of NGC 3115 compared to NGC 1399 in all inspected cases, indicating a more pronounced dip between the two color distribution peaks for the first GCS. The dip statistics, reported in last column of Table 3 , does not find a dip in the $(g-i)$ color for the GCs in first annulus of NGC 1399.

Figure 9 shows the color-color relation (CCR hereafter) for the selected GC candidates obtained in each one of the five annuli analyzed above.

For each annulus we derived a linear fit to the CCR (shown with dashed red lines in Fig. 9), and a fourth-degree polynomial fit (solid curves in various shades of blue). Only the color interval spanned by real sources is plotted for each radius. In the figure we plot two cases: in the first case, linear and polynomial fits are derived from only the GC candidates in the annulus (left panels a in the figure) and, in the second case, all GC candidates inside the given outer radius are used for the fits (right panels b). For sake of clarity, the CCR of the annuli beyond the first are shifted by multiples of $+0.5 \mathrm{mag}$ in $(g-i)$ color.

In each panel, we draw a sixth linear and polynomial fit obtained from all candidates beyond the background radius (assuming circular shape, left plots panel a; green dotted line for polynomial fit and red dashed line for the linear fit) and from all GC candidates on the frame (right plots, panel b).

The same analysis is carried out for NGC 3115 and NGC 1399 GCs with the only difference that the radii adopted have half the angular size for the latter, for the reasons described above.

Some differences between the two targets appear more pronounced for the innermost annuli, where the GC density is higher and hence the role of residual contamination is smaller. The visual impression of the difference between the two GCSs is even more evident in Fig. 10. The first two panels in the figure (from left) overplot the CCRs running mean area for NGC 3115 (light gray areas) and for NGC 1399 (dark gray). The GC candidates in the labeled annulus are shown in panel a, all candidates within the $i$ th annulus in panel $\mathrm{b}$. The right two panels in the figure plot the fourth-degree fits for same data with solid blue lines for NGC 3115 and dotted blue lines for NGC 1399. The data for background regions are shown with green areas/lines, according to the symbols in the panel ${ }^{16}$.

Consistent with visual impression, the Spearman correlation coefficient $r_{x y}$ - a nonparametric measure of statistical dependence between two variables (Spearman 1904) -, reported in Table 4 for the $(u-i)-(g-i)$ correlation, is in all cases larger for NGC 3115 than for NGC 1399 at a given annulus ${ }^{17}$.

16 The CCR for NGC 3115 extend to bluer colors than NGC 1399, mostly due to our choice of using annuli of similar linear sizes for the two galaxies. As shown in Fig. 8, the outermost annuli used for NGC 1399 do not extend to the farthest intergalactic regions that are richer in blue GCs. Furthermore, because of the much larger mass of NGC 1399 with respect to NGC 3115, a larger portion of red GCs is expected at similar physical sizes (Peng et al. 2006).

17 Because of the presence of a strong blue tilt in the GC population of NGC 1399 (Harris et al. 2006; Mieske et al. 2010), we tested the robustness of the differences between the relative shape of CCRs in the two galaxies vs. changes of the bright magnitude cut. In spite of the reduced number of GCs selected, placing the bright magnitude limit $m_{\text {bright }}$ at $2 \sigma$ or $1 \sigma$ brighter than the turnover magnitude, rather than $4 \sigma$, did not change the result, i.e., a higher degree of nonlinearity for NGC 1399 CCRs is found in both tests. 

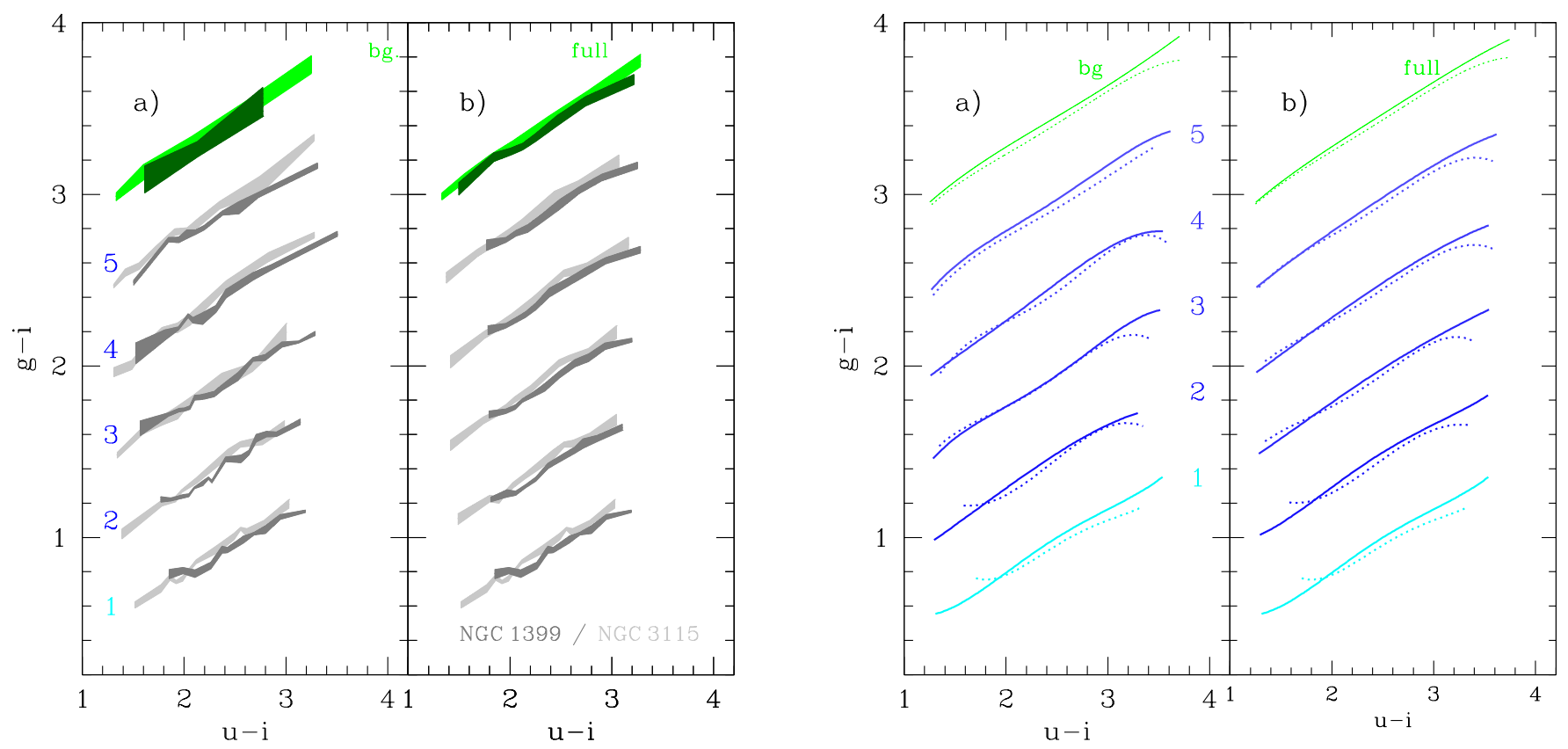

Fig. 10. Left panel: as in Fig. 9, except that both galaxies are plotted together and only the areas identified by the running median are shown. The width of the shaded areas is given by the standard deviation of the mean. Darker colors refer to NGC 1399; light gray and green to NGC 3115 . Right panel: as in Fig. 9, except that the polynomial fits for the CCRs of NGC 3115 (solid lines) are superposed on NGC 1399 (dotted lines).

Table 4. Spearman correlation coefficients for the $(u-i)-(g-i)$ relation.

\begin{tabular}{|c|c|c|}
\hline \multicolumn{3}{|c|}{$r_{x y}$ in single annuli } \\
\hline 1 & 0.940 & 0.887 \\
\hline 2 & 0.943 & 0.928 \\
\hline 3 & 0.943 & 0.931 \\
\hline 4 & 0.959 & 0.921 \\
\hline 5 & 0.959 & 0.930 \\
\hline bg & 0.958 & 0.927 \\
\hline \multicolumn{3}{|c|}{$r_{x y}$ incremental sample } \\
\hline 1 & 0.940 & 0.887 \\
\hline 2 & 0.941 & 0.912 \\
\hline 3 & 0.942 & 0.921 \\
\hline 4 & 0.947 & 0.922 \\
\hline 5 & 0.951 & 0.925 \\
\hline All & 0.960 & 0.931 \\
\hline
\end{tabular}

Overall, our results show that the CCRs of the two galaxies are very similar in general but have some differences. In spite of the identical ranges for color selections, the running mean in Figs. 9-10 shows the GC candidates population in NGC 1399 is shifted toward red colors and has a different shape with respect to GCs in NGC 3115, which underlines different median properties of the two GCSs.

Assuming, as reasonable, nearly uniform old ages for both GCSs, the color differences might mark slightly larger content of metals in NGC 1399 GCSs, which is not unexpected given the much larger luminosity, and hence mass, of the Fornax cluster giant galaxy. Our analysis also supports various degrees of nonlinearity in the CCRs of the two targets with the CCRs in NGC 1399 appearing more nonlinear. Such difference is reduced for the CCRs at larger galactocentric distances, as expected, owing to the increased relative contamination from MW stars.

Since old GC colors are mainly driven by metallicity, at least one of the color-metallicity relations involved must be nonlin- ear to some degree. Hence, making it doubtful any analysis of the metallicity distribution drawn from the simplistic assumption of bimodal metallicity distributions evidenced by bimodal color distributions, i.e., by assuming linear color-metallicity relations. Nevertheless, given the dependence of the shape of the CCRs on the presence of few very red or very blue GCs, the results shown here are not robust enough to be generalized. We will further study this specific issue on other galaxies of the VEGAS sample with different masses and in different environments.

\subsection{Radial density distributions and (local) specific frequency}

The radial density profiles of GCs in both our targets out to large galactocentric distances have been thoroughly analyzed in other studies (for NGC 3115, Cantiello et al. 2015, and references therein; for NGC 1399, Dirsch et al. 2003; Bassino et al. 2006; Schuberth et al. 2010; Kim et al. 2013a).

Now we compare the two targets highlighting how, in spite of the differences emerged for two-dimensional spatial distribution and for the color distributions discussed above, some degree of homogeneity in the radial density profiles of the two GC systems appears, especially for the red GC component.

The background-corrected azimuthally averaged radial density of GC candidates in each field was fitted to a de Vaucouleurs (1948) $r^{1 / 4}$ law plus a constant background. We also fitted the $r^{1 / 4}$ profile to the blue and red GC subpopulations separately ${ }^{18}$.

The results are shown in Fig. 11. For both GCSs, our analysis confirms the results of previous similar studies on these and other targets: first, GCs have a shallower light profile than the galaxy

${ }^{18}$ We fit the surface density profiles with a Reynolds-Hubble law and a Sersic law. The improvement of the matching for the best-fitting curve to data is marginal, in particular using the Reynold-Hubble profile the inner radii of the distributions get a better match to data. However, the distributions add further parameters, i.e., degrees of freedom, to the fits, while the general trends observed do not change notably. 

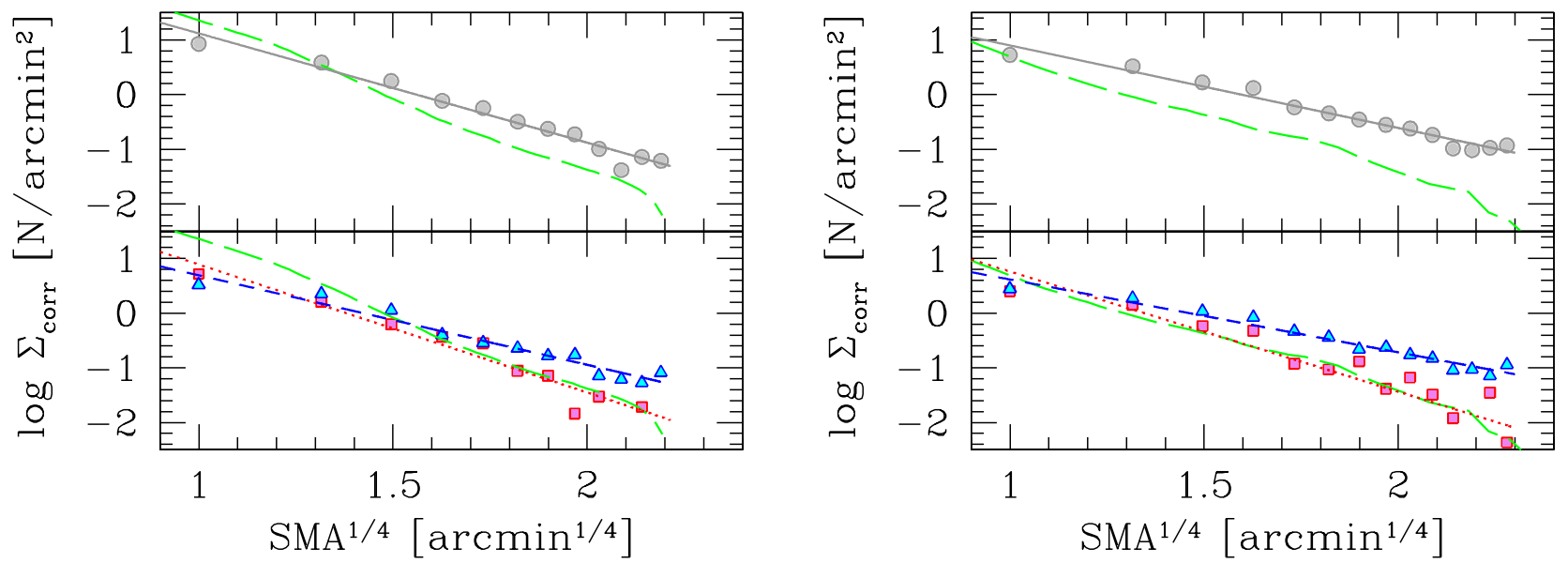

Fig. 11. Upper left panel: surface density of GC candidates in NGC 3115, corrected for background contamination, vs. galaxy semimajor axis length, SMA. The binned data are shown with gray circles, the $r^{1 / 4}$ law is fit with a solid line. The green long-dashed line denotes the $g$-band light profile from Spavone et al. (in prep.), arbitrarily scaled vertically to match the red GC density profile. Lower left panel: as upper, but separately for blue GCs (blue triangles and dashed line) and red GCs (red squares and dotted line). Right panels: as left, but for the GCs in NGC 1399.

field star light and second, the density profile of red GCs is more concentrated than that of blue GCs and also closely follows the underlying galaxy light profile.

For NGC 3115, we determined the semimajor axis where the density of GCs drops below 1 over an isodensity annulus one arcminute wide, $S M A_{1}$, in addition to the radial profiles in Fig. 11 and given the observed larger radial extent of the GC population with respect to the field stars light distribution, to obtain a rough estimate of the radius in which the GC density is negligible. Using an extrapolation to the total GC radial density profile shown in Fig. 11 (right panels) and adopting an approximate incompleteness factor of $\sim 2$ (see below), we estimated $S M A_{1} \sim 61^{\prime}$. Such a crude estimate relies on various assumptions: i) linear extrapolation to the GC density profile (from Fig. 11); ii) similarity between the geometry of galaxy light and GC distribution, which is only motivated by the comparable profiles within $\sim 25^{\prime}$; and iii) negligible contamination of the GCs beyond the assumed background radius, $r_{\mathrm{bg}}=29^{\prime}$. In comparison, the surface brightness profile drops to $\mu_{g} \sim 30 \mathrm{mag} \operatorname{arcsec}^{-2}$ at $S M A \sim 25^{\prime}$ (Spavone et al., in prep.).

A final relevant comparison between the two GCSs is the specific frequency, $S_{N}$. By definition, $S_{N}$ is a global quantity characterizing the properties of the galaxy and its host GCs. Nevertheless, we analyzed the behavior of $S_{N}$ vs. radius, $S_{N}(<r)$, obtained from the total magnitude within the given radius and total number of GCs enclosed within the same area. Because of the incompleteness, in particular for NGC 1399, to obtain absolute $S_{N}$ approximately comparable with existing literature values, we corrected the total number of enclosed GCs by a scaling factor obtained from the ratio of the GC counts within a common detection area between our study and the studies from deep imaging of the same targets (for NGC 3115 Jennings et al. 2014; Jordán et al. 2015, for NGC 1399). We found that the scaling factor for incompleteness is $\sim 2(\sim 11)$ for NGC 3115 (NGC 1399) with an estimated number of $\sim 40(\sim 150)$ unobserved GCs in the central galaxy regions. In Fig. 12, we show the results of the analysis of the local $S_{N}(<r)$, which are derived using the total number of GCs and total magnitude within a given radius in place of $N_{\text {tot }}$ and $M_{V}^{\text {tot }}$. We corrected the determinations of $S_{N}(<r)$ for the rough incompleteness factors given above, although we are interested in the relative comparison of the two GC systems.
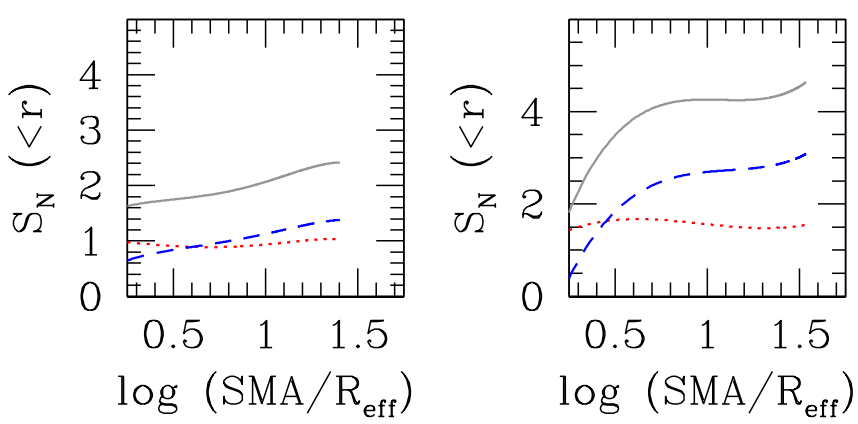

Fig. 12. Left panel: local value of the specific frequency of GCs, $S_{N}(<r)$, for NGC 3115. The solid gray line shows the full GC population, the blue dashed and red dotted lines show the local $S_{N}^{\text {blue }}(<r)$ and $S_{N}^{\text {red }}(<r)$ for blue and red GCs separately. Right panel: as left, but for NGC 1399 .

As for the asymptotic limit of $S_{N}$, we find the specific frequency for NGC 1399 is a factor of $\sim 2$ higher than NGC 3115, mainly because of extra blue GCs.

The $S_{N}(<r)$ for the total GC population (gray lines in left panel for NGC 3115, right panel for NGC 1399) reveals very different radial trends: relatively smooth for NGC 3115, and rapidly increasing then smooth at $S M A \geq 10^{\prime}$ for NGC 1399 , with possible signs of a further increase at larger radii.

It is instructive to inspect the local specific frequency for the blue and red GCs, $S_{N}^{\text {blue }}(<r)$ and $S_{N}^{\text {red }}(<r)$, separately; these are shown with dashed blue and dotted red lines in Fig. 12. The estimated local frequency for the red GCs is nearly constant over the range of inspected radii for both galaxies. Furthermore, the similarity of the observed $S_{N}^{\text {red }}(<r)$ between the two galaxies implies that, contrary to expectation, NGC 3115 has a relatively high proportion of red GCs than NGC 1399. The blue GC component, hence, is the dominant, although is not the only cause of the difference between the $S_{N}(<r)$ of the total GC population. The fraction of blue GCs relative to field stars in NGC 3115 grows smoothly and steadily with galaxy radius, while it shows a quasi-inflection point in NGC 1399, where the value is basically constant at around $\sim 10^{\prime}$ and then shows signs of a further increase at larger radii. 


\section{Discussion}

\subsection{Differences and similarities}

It is an interesting question to ask to what degree the level of galaxy clustering and the inherent hierarchical assembly affects the observed GCSs properties (see, e.g., Blakeslee et al. 1997; Côté et al. 1998; Blakeslee 1999; Peng et al. 2008; Hudson et al. 2014). We started to answer this question by presenting the data for two very dissimilar targets with the purpose of further extending the study as the sample of galaxies observed within VEGAS and the area covered by FDS increase.

If compared to the GC population in NGC 1399, the GCs in NGC 3115 formed and evolved in a very isolated environment. The present status of the GC system, then, is less contaminated by the evolutionary effects active on a central cluster galaxy, and there have been much fewer changes to the original properties of the system, which could be considered more pure than for the GCS in NGC 1399. Our work indicates that the GC systems of NGC 3115 and NGC 1399 are markedly different in some aspects, while sharing similar behavior on some others.

Based on the surface density maps and radial profiles of field stars and GCs, we find that both stellar components in NGC 3115 are well thermalized with each other. The observed similarity between the red GC density profile and galaxy stellar light profile out to $\sim 25^{\prime}$, the resemblance of the geometry of the distribution of red and blue GCs with the galaxy isophotes, the combined observational evidence that blue GCs follow the X-ray profile of the galaxy (Forte et al. 2005; Forbes et al. 2012) and that X-rays trace the dark matter in the galaxy point toward a one-to-one correlation between galaxy light and GCS, suggesting a very closely related formation history of the systems. Furthermore, the analysis of resolved stars in the galaxy based on HST data of three fields between 7 and 21 effective radii, has shown that the densities of halo stars and GCs also appear to decrease in a similar fashion with increasing radius, providing evidence of the common formation of blue GCs and halo stars (Peacock et al. 2015).

For NGC 1399, we confirm and corroborate the previous observations for markedly different distribution of blue and red GCs. Red clusters are closely associated with the spheroid of the central giant galaxy and other bright galaxies angularly close to NGC 1399. Blue GCs are more diffuse and elongated along the east-west direction of the cluster. On the eastern side the angular position in the sky of such a diffuse blue GC component is not obviously associated with any bright galaxy; the intergalactic nature of the blue GC cloud does not tell much about its origin, whether the GCs come from primordial mini dark-matter halos, or are stripped from local satellites or from any other process. Nevertheless, a later accretion of blue GCs from local dwarfs would explain the observed differences of azimuthal distributions with respect to the red GCs. West of NGC 1399, some evidence from the bridges of GCs toward NGC 1387 and NGC 1381 suggest that those GCs are stripped from the halos of their host galaxies.

The GC color distributions and color-color relations of the two galaxies also present differences worth highlighting. Both galaxies share the property of a markedly bimodal color distribution in $(u-i)$ and $(g-i)$, a common property of bright galaxies. While for NGC 3115 there is universal consensus for a bimodal metallicity distribution generating the observed color bimodality, the case of NGC 1399 is less obvious. By comparing the CCRs for the sources in approximately equivalent spatial intervals of the two galaxies, we observe non-negligible differences. The CCRs for NGC 1399 annuli appear shifted toward redder $(u-i)$ for a given $(g-i)$ and these CCRs are slightly, but no- ticeably, more nonlinear than in NGC 3115. Assuming all GCs are approximately equally old, it is easy to associate the redder colors with on average higher metal content. The slightly different shapes of the CCRs might be related either to the presence of age structures, such as age gaps, or to other physical parameters affecting the horizontal branch morphology, for example (e.g., helium content; D'Antona et al. 2005; Gratton et al. 2010). The efficiency of galaxy and GCSs cannibalism in the core of Fornax for NGC 1399 may have led to a stratification of GC subcomponents, producing as a result a collection of GCs with a broad range of metallicity and no distinctive age structure; instead, there may be a gap between the ages of metal-rich and metal-poor populations which appear more distinctly separated for NGC 3115.

Together with previous results from Blakeslee et al. (2012), the differences between the two sets of CCRs might be pointing out a possible role of nonlinear color-metallicity relations in shaping the observed color distribution in NGC 1399. The complex nature of the GCS around this bright galaxy hampers the simple equivalence of color-bimodality $\equiv$ metallicitybimodality, hence raising key questions: how frequently is the color bimodality we observe due to real metallicity bimodality? Or, viceversa, how often is an artifact generated by some projection effect on a parent distribution that would otherwise have any other shape (multimodal, smooth, and broad, asymmetric, etc.)?

The MW GCS is another system we know has a wellknown bimodal $[\mathrm{Fe} / \mathrm{H}]$ distribution. If placed at the distance of $10 / 20 \mathrm{Mpc}$, i.e., at the distance of NGC 3115/Fornax cluster, the MW with its bright companion M 31 at $\sim 1.15^{\circ} / 2.3^{\circ}-$ and a number of companions brighter than $M_{V} \sim-15$ mag - appears in an environment with intermediate density that is not as isolated as NGC 3115 nor as companion rich as NGC 1399. Hence, it would be tempting to look for purely bimodal $[\mathrm{Fe} / \mathrm{H}]$ distributions in GCS within galaxies in small groups of galaxies, such as the MW, or in isolated galaxies, such as NGC 3115. However, the case of the GCS in M31 is not as obvious as the MW, with a multimodal rather than bimodal $[\mathrm{Fe} / \mathrm{H}]$ distribution (Galleti et al. 2009; Caldwell et al. 2011). NGC 5128 and NGC 4494 are similar cases of galaxies in small groups or isolated from large groups with well-studied GCSs and bimodal color distributions, where the spectroscopically derived GCS metallicity shows complex distributions with perhaps three peaks (Beasley et al. 2008; Woodley et al. 2010; Foster et al. 2011; Usher et al. 2012). The complex GCS for these three large galaxies gives evidence that they had more active formation histories than the MW. Nevertheless, given the locations in sparsely populated regions, their formation histories were not as turbulent as those of cluster ellipticals, such as NGC 1399 (Puzia et al. 2014; Webb et al. 2016)

A further difference we observed between NGC 3115 and NGC 1399 is related to the local specific frequency $S_{N}(<r)$, i.e., the $S_{N}$ value obtained from the magnitude and GC number integrated within a given radius, a relative comparison largely insensitive to any GC magnitude incompleteness. We observe a steady increase of $S_{N}(<r)^{\text {blue }}$ for the blue GCs in NGC 3115, which is not unexpected given the way $S_{N}$ is defined, because the blue GC density profile is more extended than the galaxy light. In the case of NGC 1399 we first observe a steep increase of $S_{N}(<r)^{\text {blue }}$ followed by a relatively flat region and then a possible further rise of the local specific frequency at large galactocentric radii. Although our analysis of $S_{N}(<r)$ is not quantitatively as refined as the surface brightness analysis presented in Iodice et al. (2016), the flat region we identify in the local specific frequency diagram matches with the break radius found by 
Iodice et al. at $r \sim 10^{\prime}$, which marks the transition between the galaxy and its stellar halo fading into the intracluster light.

The behavior of the local specific frequency of red GCs, $S_{N}^{\text {red }}(<r)$, appears very similar between the GC systems of the two galaxies. The step-wise behavior of red GCs and galaxy field stars is commonly assumed to be evidence of their common formation history (Forbes et al. 2012; Kartha et al. 2016). Furthermore, our results on i) the nearly flat $S_{N}^{\text {red }}(<r)$ out to $\sim 25$ effective radii; ii) the similar in behavior of red GCs and the galaxy with radius, and within the limits of the approximate estimate of the magnitude incompleteness, and iii) the close absolute values of $S_{N}^{\text {red }}(<r)$ despite the large differences existing between the two galaxies compared here (we find $S_{N}^{\text {red }}(<r)$ ranging between $\sim 1$ and $\lesssim 1.7$ for both galaxies), imply a deeper similarity not only of the red GCs and galaxy field stars within the galaxy, but also from one galaxy to the other, even in extremely different environments. We will further inspect and discuss the appearance of such similarity with the future VEGAS and FDS datasets.

A final noteworthy similarity of the two galaxies is the crossing radius of the $S_{N}^{\text {blue }}(<r)$ and $S_{N}^{\text {red }}(<r)$ curves, which appear very similar in the adopted normalized distance units.

\subsection{Cosmological context}

The literature on GCSs formation and evolution, in connection with the history of galaxy formation and evolution, is very rich. As already mentioned in the introductory section, in a cosmological context the scenarios proposed for GC formation can be broadly classified into two families. In one case the blue and red GCs have an age gap of $\lesssim 2$ Gyr and in the second they are approximately coeval.

In the age-gap case, there seems to be consensus toward a scenario in which the earliest stellar populations in a protogalaxy, halo field stars, and metal-poor GCs form early with GCs growing from the rare density peaks of the primordial dark matter field, which is a process interrupted by cosmic reionization (Diemand et al. 2005; Moore et al. 2006). In this context, blue GCs would have nearly constant abundances with small deviations caused by different local reionization epochs, an expectation that is compatible with the small color scatter of blue GCs (Peng et al. 2006; Brodie \& Strader 2006; Harris et al. 2006).

The metal-rich GC population subsequently formed after a dormant period in the dissipational processes that built up the bulk starlight of the massive seed galaxies with mean $[\mathrm{Fe} / \mathrm{H}]$ of the system following a mass-metallicity relation.

Other authors, still within the blue and red GCs age-gap scenario, adopted slightly different schemes more or less explicitly rejecting the role of reionization in stopping the formation of metal poor clusters (see Forbes et al. 2015, and references therein). In general, any scenario proposing different mechanisms and/or epochs of formation for the two GC subpopulations should explain the strong similarities of the two subpopulations.

In the family of models in which the red and blue GCs are coeval, Côté et al. (1998, 2000, 2002) proposed GCSs formation from dissipationless hierarchical growth. The metal-poor GC component is accreted to the massive seed galaxy through mergers or tidal stripping. Also in this scenario the blue GCs are predicted to have common properties with the galaxy halo. The metal-rich clusters represent the intrinsic GC population of massive galaxy seeds.

To date, observational evidence does not allow us to firmly establish the existence of an age gap between the blue and red GCs, not even in the MW GC system (Strader et al. 2005; Marín-Franch et al. 2009; VandenBerg et al. 2013). Furthermore, as discussed in previous sections, the last decade has seen increasing interest toward the projected bimodality, i.e., the possibility that a given fraction of the observed color bimodality of extragalactic GCSs is not necessarily a one-to-one match of the $[\mathrm{Fe} / \mathrm{H}]$ distribution. Hence, currently there is no consensus about the existence of age gaps between GC subpopulations and on the ubiquitousness of metallicity bimodality. drawn

Summarizing from above, two approximate scenarios can be

In the first case, blue metal-poor GCs form at the very early stages in galaxy evolution from primordial density fluctuations. The formation process is spatially very uniform and is halted by a spatially extended event taking place on a relatively short timescale, such as cosmic reionization. Red metal-rich GCs form later in a dissipational or dissipative process that builds up the red GCs together with the bulk of the host galaxy starlight.

In the second case, all GCs are coeval, form from primordial fluctuations of the dark matter density field, and mean abundances of the forming star clusters are directly affected by the depth of the potential well of the protogalaxy. Then, the dissipationless accretion of blue GCs from dwarf galaxies completes the shaping of the present day GCSs.

In both scenarios, there are two requirements. First, the blue GCs share common history with the galaxy halo, avoid feedback processes, and thereby explain the relatively uniform properties of GCs and their strong ties to the galaxy dark matter halo properties, such as the essentially constant mass ratio $\eta=M_{\mathrm{GCS}} / M_{\text {halo }} \sim 4 \times 10^{-5}$ (Hudson et al. 2014) (see also Peng et al. 2008; Spitler \& Forbes 2009; Georgiev et al. 2010). Second, red GCs share common history with the bulk starlight of the massive seed galaxy.

The results we presented in this paper, on the comparison between the GCSs of NGC 3115 and NGC1399, are compatible with both such broadview scenarios.

In either the single age or age-gap case, the GCs in the isolated NGC 3115 had evolved after the last GC star forming episode without much interaction with other massive companion galaxies. Even supposing the latest GC formation ages to $\sim 10$ Gyr (VandenBerg et al. 2013; Forbes et al. 2015), the blue and red GCs, dark matter halo, and galaxy stellar systems had a long common stage of evolution, which was sufficient to virialize all the dark, stellar, and GC matter components. The presence of a clear $[\mathrm{Fe} / \mathrm{H}]$ bimodality, hence of a well-defined, metal-rich GC component with mean properties clearly separated from the metal-poor component, could be the result of the presence of a unique deep potential well, in which the red GCs formed and evolved in a common epoch with the blue metal-poor GCs residing in the halo; or this presence could result from multiple nearly same-sized lower mass wells, each with similar red GCs, summing up in the present-day GC populations.

Also the red GCs in NGC 1399 share common properties with the galaxy field stars. As for blue GCs, they appear to fade into the cluster halo density distribution, a property common to other galaxy clusters as well (Peng et al. 2010; Durrell et al. 2014). The GC metallicity bimodality appears less obvious than in NGC 3115, as would be expected in a picture of hierarchical growth in a dense environment such as Fornax. The accretion of red GCs associated with galaxy progenitors with different masses, hence with a different position of the red GC metallicity peak, generates the superposition of metallicity distributions; after the addition of the more uniform blue GC component, 
this superposition is hard to reconcile with a simple bimodal $[\mathrm{Fe} / \mathrm{H}]$ pattern.

A plausible scenario that seems to emerge is that the asymmetric blue GC distribution visible in Fig. 6 is connected to the large-scale cluster potential well (the cluster halo, one of the three X-ray components detected by Paolillo et al. 2002) preceding the bulk of the cluster X-ray emitting gas, which is slowed by ram pressure stripping due to the interaction with the halo of the NGC 1316 subgroup. Red GCs instead follow the stellar mass density profiles of the individual galaxies more closely and, in particular, the galactic halo component from Paolillo et al. (2002) in the NGC 1399 case.

\section{Summary and conclusions}

In this study we compared the properties of the GCSs in NGC 3115 and NGC 1399 as derived from the analysis of one square degree $u-, g$, and $i$-band images of the fields centered on the galaxies, taken with the VST telescope as part of the VEGAS and FDS surveys.

The two galaxies analyzed are very differently from each other. NGC 3115 is one of the closest lenticular galaxies, at $\sim 10 \mathrm{Mpc}$; this galaxy is very isolated with only one close companion galaxy brighter than $M_{V} \sim-15$ mag within a projected area of $100 \mathrm{deg}^{2}$.

NGC 1399, located near the dynamical center of the Fornax cluster, is the second brightest early-type galaxy of the cluster located in a densely populated region hosting 43 galaxies brighter than $M_{V} \sim-15$ mag over a projected area of $100 \mathrm{deg}^{2}$.

For the two galaxies we analyzed the surface density maps, color distributions, and radial density profiles of GC candidates, selecting the list of candidates using as reference the morphophotometric properties and colors of confirmed GCs available in the literature. Our main conclusions are the following:

1. The field stellar light and GC density maps of NGC 3115 closely follow each other in terms of position of the center, elongation, and inclination.

2. For NGC 1399, GC density maps confirm the known presence of substructures. The GC overdensity covers a large portion of inspected area, providing supporting evidence in favor of its intergalactic nature. Globally, the morphology of the overdensity is asymmetric with an elongated east-west shape, and rich with substructures already discussed by, for example, D'Abrusco et al. (2016). On large scales, 30', we do not find obvious correspondence between the GC surface density and the cluster-halo X-ray component first discussed by Paolillo et al. (2002), which has a southwest to northeast elongation. In particular, we observe a spatial offset in the NGC 1399 GC centroid with respect to galaxy field stellar light that does not match the X-ray density contours. On smaller angular scales $\left(\lesssim 10^{\prime}\right) \mathrm{X}$-ray and GC contours appear more similar.

3. For both galaxies the GC system has larger spatial extent compared to galaxy light. For NGC 3115, the semimajor axis where the density of GCs drops below 1 over an isodensity annulus one arcminute wide is $S M A_{1} \sim 61^{\prime}$, for comparison the surface brightness profile of field stars drops to $\mu_{q} \sim 30 \mathrm{mag} \operatorname{arcsec}^{-2}$ at $S M A \sim 25^{\prime}$.

4. The shape of blue and red GC maps for NGC 3115 do not differ much from each other.

5. The blue and red GC surface density maps for NGC 1399 are notably different from each other: red GCs are mostly concentrated around bright galaxies and blue GCs occupy the entire observed region and generate the observed eastwest overdensity.

6. Both galaxies show bimodal color distributions in $(u-i)$ and $(g-i)$ and are more prominent in $(u-i)$, especially for NGC 3115

7. The color-color relations of the two galaxies do not overlap. The GC candidate population in NGC 1399 is shifted toward red and has CCRs that are slightly different with respect to GCs in NGC 3115, which is quantitatively more nonlinear.

8. Our results support existing results on GC metallicity bimodality as the main cause of the bimodal color distribution in NGC 3115. The case of NGC 1399 is less obvious, as would be expected in a dense environment like Fornax.

9. The azimuthally averaged radial density profile of GC candidates, for both galaxies, reaches larger galactocentric radii than the field stellar light distribution. Moreover, the density profile of red GCs is more concentrated than that of blue GCs and follows the underling galaxy light profile.

10. The local specific frequency for the total GC populations is notably different for the two galaxies. For NGC $1399 S_{N}$ is a factor of $\sim 2$ higher than that for NGC 3115, mainly because of extra blue GCs. By inspecting the local specific frequency for red GCs, the radial trend and absolute values of $S_{N}^{\text {red }}(<r)$ appear very similar from one galaxy to another. Such similarity implies a deeper similarity not only of the red GCs and galaxy field stars within the galaxy, but also from one galaxy and the other, even in extremely different environments.

11. Whether or not red and blue GCs are coeval, our observations confirm, and further strengthen, the need for blue GCs to share a common history with the galaxy dark matter halo, and for red GCs to be more closely bound to the galactic stellar field component.

12. Overall, our analysis shows that field stars and GCs in NGC 3115 are well thermalized with each other. In the isolated host environment the blue and red GCs, dark matter halo, and galaxy stellar systems had a long common stage of evolution that was sufficiently long to virialize all the components.

13. For NGC 1399 the red GCs share common properties with the galaxy field stars, while blue GCs appear either associated with the galaxy halo or to fade into an intracluster $\mathrm{GC}$ component. We speculate that the emerging pattern is that the two Fornax subclusters are falling toward each other, with the galaxies and the (halo) GCSs moving ahead of the gas component because of their noncollisional nature.

Additional wide-field imaging studies of GC populations for a large number of galaxies of different masses and in various environments, together with new constraints on the relative ages of various GC subpopulations (if any), along with revised formation models free from unneeded observational constraints, will be important in assessing the formation process of GCs and of all the galaxy components sharing common evolutionary paths with GCs. As the VEGAS and FDS surveys proceed, more multiband imaging data over large areas for early-type galaxies will be available; this resource is extremely useful for this purpose.

Acknowledgements. The data reduction for this work was carried out with the computational infrastructures of the VST Center at Naples (VSTceN). We gratefully acknowledge INAF for financial support to the VSTceN. We acknowledge the usage (Makarov et al. 2014) of the HyperLeda database, http: //leda.univ-lyon1.fr. This research has made use of the NASA Astrophysics Data System Bibliographic Services, the NASA Extragalactic Database, the SIMBAD database, operated at CDS, Strasbourg, and of Aladin sky atlas developed at CDS, Strasbourg Observatory, France. Based on observations collected at the European Organisation for Astronomical Research in the 
Southern Hemisphere under ESO programs 088.B-4012(A), 090.B-0414(B), 090.B-0414(C), 090.B-0414(D), 092.B-0623(C), 092.B-0623(D), and 094.B0496(A). Michele Cantiello acknowledges John L. Tonry for providing the code colmerge, used to match the photometric tables, and Lucia Panico for useful discussions.

\section{References}

Alamo-Martínez, K. A., Blakeslee, J. P., Jee, M. J., et al. 2013, ApJ, 775, 20 Alves-Brito, A., Hau, G. K. T., Forbes, D. A., et al. 2011, MNRAS, 417, 1823 Arnold, J. A., Romanowsky, A. J., Brodie, J. P., et al. 2011, ApJ, 736, L26 Ashman, K. M., \& Zepf, S. E. 1992, ApJ, 384, 50

Bassino, L. P., Cellone, S. A., Forte, J. C., \& Dirsch, B. 2003, A\&A, 399, 489 Bassino, L. P., Faifer, F. R., Forte, J. C., et al. 2006, A\&A, 451, 789

Beasley, M. A., Baugh, C. M., Forbes, D. A., Sharples, R. M., \& Frenk, C. S. 2002, MNRAS, 333, 383

Beasley, M. A., Bridges, T., Peng, E., et al. 2008, MNRAS, 386, 1443

Bekki, K., Forbes, D. A., Beasley, M. A., \& Couch, W. J. 2003, MNRAS, 344 1334

Bekki, K., Yahagi, H., Nagashima, M., \& Forbes, D. A. 2008, MNRAS, 387, 1131

Bergond, G., Athanassoula, E., Leon, S., et al. 2007, A\&A, 464, L21

Bertin, E., \& Arnouts, S. 1996, A\&AS, 117, 393

Blakeslee, J. P. 1999, AJ, 118, 1506

Blakeslee, J. P., Tonry, J. L., \& Metzger, M. R. 1997, AJ, 114, 482

Blakeslee, J. P., Jordán, A., Mei, S., et al. 2009, ApJ, 694, 556

Blakeslee, J. P., Cantiello, M., \& Peng, E. W. 2010, ApJ, 710, 51

Blakeslee, J. P., Cho, H., Peng, E. W., et al. 2012, ApJ, 746, 88

Bridges, T. J., Hanes, D. A., \& Harris, W. E. 1991, AJ, 101, 469

Brodie, J. P., \& Strader, J. 2006, ARA\&A, 44, 193

Brodie, J. P., Usher, C., Conroy, C., et al. 2012, ApJ, 759, L33

Brodie, J. P., Romanowsky, A. J., Strader, J., et al. 2014, ApJ, 796, 52

Caldwell, N., Schiavon, R., Morrison, H., Rose, J. A., \& Harding, P. 2011, AJ, 141,61

Cantiello, M., \& Blakeslee, J. P. 2007, ApJ, 669, 982

Cantiello, M., Blakeslee, J. P., \& Raimondo, G. 2007, ApJ, 668, 209

Cantiello, M., Grado, A., Blakeslee, J. P., et al. 2013, A\&A, 552, A106

Cantiello, M., Blakeslee, J. P., Raimondo, G., et al. 2014, A\&A, 564, L3

Cantiello, M., Capaccioli, M., Napolitano, N., et al. 2015, A\&A, 576, A14

Capaccioli, M., Held, E. V., \& Nieto, J.-L. 1987, AJ, 94, 1519

Capaccioli, M., Spavone, M., Grado, A., et al. 2015, A\&A, 581, A10

Carretta, E., Bragaglia, A., Gratton, R. G., et al. 2009, A\&A, 505, 117

Chies-Santos, A. L., Larsen, S. S., Kuntschner, H., et al. 2011, A\&A, 525, A20

Cho, H., Blakeslee, J. P., Chies-Santos, A. L., et al. 2016, ApJ, 822, 95

Cohen, J. G., Blakeslee, J. P., \& Ryzhov, A. 1998, ApJ, 496, 808

Cohen, J. G., Blakeslee, J. P., \& Côté, P. 2003, ApJ, 592, 866

Cook, D. O., Dale, D. A., Johnson, B. D., et al. 2014, MNRAS, 445, 890

Côté, P. 1999, AJ, 118, 406

Côté, P., Marzke, R. O., \& West, M. J. 1998, ApJ, 501, 554

Côté, P., Marzke, R. O., West, M. J., \& Minniti, D. 2000, ApJ, 533, 869

Côté, P., McLaughlin, D. E., Hanes, D. A., et al. 2001, ApJ, 559, 828

Côté, P., West, M. J., \& Marzke, R. O. 2002, ApJ, 567, 853

D’Abrusco, R., Cantiello, M., Paolillo, M., et al. 2016, ApJ, 819, L31

D’Antona, F., Bellazzini, M., Caloi, V., et al. 2005, ApJ, 631, 868

de Vaucouleurs, G. 1948, Annales d'Astrophysique, 11, 247

de Vaucouleurs, G., de Vaucouleurs, A., Corwin, Jr., H. G., et al. 1991

Third Reference Catalogue of Bright Galaxies, Vol. 1-3 (Berlin, Heidelberg,

New York: Springer-Verlag)

Diemand, J., Madau, P., \& Moore, B. 2005, MNRAS, 364, 367

Dirsch, B., Richtler, T., Geisler, D., et al. 2003, AJ, 125, 1908

Drinkwater, M. J., Gregg, M. D., \& Colless, M. 2001, ApJ, 548, L139

Durrell, P. R., Côté, P., Peng, E. W., et al. 2014, ApJ, 794, 103

Ferguson, H. C. 1989, AJ, 98, 367

Firth, P., Drinkwater, M. J., Evstigneeva, E. A., et al. 2007, MNRAS, 382, 1342

Forbes, D. A., Brodie, J. P., \& Grillmair, C. J. 1997, AJ, 113, 1652

Forbes, D. A., Grillmair, C. J., Williger, G. M., Elson, R. A. W., \& Brodie, J. P. 1998, MNRAS, 293, 325

Forbes, D. A., Beasley, M. A., Brodie, J. P., \& Kissler-Patig, M. 2001, ApJ, 563, L143

Forbes, D. A., Ponman, T., \& O’Sullivan, E. 2012, MNRAS, 425, 66

Forbes, D. A., Pastorello, N., Romanowsky, A. J., et al. 2015, MNRAS, 452, 1045

Forte, J. C., Faifer, F., \& Geisler, D. 2005, MNRAS, 357, 56

Foster, C., Spitler, L. R., Romanowsky, A. J., et al. 2011, MNRAS, 415, 3393

Galleti, S., Bellazzini, M., Buzzoni, A., Federici, L., \& Fusi Pecci, F. 2009 A\&A, 508, 1285

Gebhardt, K., \& Kissler-Patig, M. 1999, AJ, 118, 1526
Geisler, D., \& Forte, J. C. 1990, ApJ, 350, L5

Geisler, D., Lee, M. G., \& Kim, E. 1996, AJ, 111, 1529

Georgiev, I. Y., Puzia, T. H., Goudfrooij, P., \& Hilker, M. 2010, MNRAS, 406, 1967

Grado, A., Capaccioli, M., Limatola, L., \& Getman, F. 2012, Mem. Soc. Astron. It. Suppl., 19, 362

Gratton, R., Sneden, C., \& Carretta, E. 2004, ARA\&A, 42, 385

Gratton, R. G., Carretta, E., Bragaglia, A., Lucatello, S., \& D’Orazi, V. 2010, A\&A, 517, A81

Griffen, B. F., Drinkwater, M. J., Thomas, P. A., Helly, J. C., \& Pimbblet, K. A. 2010, MNRAS, 405, 375

Grillmair, C. J., Freeman, K. C., Bicknell, G. V., et al. 1994, ApJ, 422, L9

Grillmair, C. J., Forbes, D. A., Brodie, J. P., \& Elson, R. A. W. 1999, AJ, 117, 167

Hargis, J. R., \& Rhode, K. L. 2014, ApJ, 796, 62

Harris, W. E. 1991, ARA\&A, 29, 543

Harris, W. E. 2001, in Saas-Fee Advanced Course 28: Star Clusters (Berlin, Heidelberg: Springer-Verlag)

Harris, W. E., \& Hanes, D. A. 1987, AJ, 93, 1368

Harris, W. E., \& Harris, G. L. H. 2002, AJ, 123, 3108

Harris, W. E., \& van den Bergh, S. 1981, AJ, 86, 1627

Harris, W. E., Whitmore, B. C., Karakla, D., et al. 2006, ApJ, 636, 90

Harris, W. E., Harris, G. L. H., \& Alessi, M. 2013, ApJ, 772, 82

Harris, W. E., Ciccone, S. M., Eadie, G. M., et al. 2017, ApJ, 835, 101

Hartigan, J. A., \& Hartigan, P. M. 1985, The Annals of Statistics, 13, 70

Hilker, M. 2015, IAU General Assembly, 22, 2215721

Hilker, M., Infante, L., \& Richtler, T. 1999, A\&AS, 138, 55

Hudson, M. J., Harris, G. L., \& Harris, W. E. 2014, ApJ, 787, L5

Iodice, E., Capaccioli, M., Grado, A., et al. 2016, ApJ, 820, 42

Iodice, E., Spavone, M., Cantiello, M., et al. 2017, ApJ, 851, 75

Janssens, S., Abraham, R., Brodie, J., et al. 2017, ApJ, 839, L17

Jedrzejewski, R. I. 1987, MNRAS, 226, 747

Jennings, Z. G., Strader, J., Romanowsky, A. J., et al. 2014, AJ, 148, 32

Jordán, A., Peng, E. W., Blakeslee, J. P., et al. 2015, ApJS, 221, 13

Kartha, S. S., Forbes, D. A., Alabi, A. B., et al. 2016, MNRAS, 458, 105

Kim, H.-S., Yoon, S.-J., Sohn, S. T., et al. 2013a, ApJ, 763, 40

Kim, S., Yoon, S.-J., Chung, C., et al. 2013b, ApJ, 768, 138

Kissler-Patig, M., Richtler, T., \& Hilker, M. 1996, A\&A, 308, 704

Kissler-Patig, M., Kohle, S., Hilker, M., et al. 1997, A\&A, 319, 470

Kissler-Patig, M., Brodie, J. P., Schroder, L. L., et al. 1998, AJ, 115, 105

Kissler-Patig, M., Grillmair, C. J., Meylan, G., et al. 1999, AJ, 117, 1206

Kravtsov, A. V., \& Gnedin, O. Y. 2005, ApJ, 623, 650

Kron, R. G. 1980, ApJS, 43, 305

Kruijssen, J. M. D. 2014, Class. Quantum Grav., 31, 244006

Kundu, A., \& Whitmore, B. C. 2001, AJ, 121, 2950

Kundu, A., Zepf, S. E., Hempel, M., et al. 2005, ApJ, 634, L41

Landolt, A. U. 1992, AJ, 104, 340

Larsen, S. S., Brodie, J. P., Huchra, J. P., Forbes, D. A., \& Grillmair, C. J. 2001, AJ, 121, 2974

Li, H., \& Gnedin, O. Y. 2014, ApJ, 796, 10

Liu, C., Peng, E. W., Jordán, A., et al. 2011, ApJ, 728, 116

Makarov, D., Prugniel, P., Terekhova, N., Courtois, H., \& Vauglin, I. 2014, A\&A, 570, A13

Marín-Franch, A., Aparicio, A., Piotto, G., et al. 2009, ApJ, 694, 1498

Mentz, J. J., La Barbera, F., Peletier, R. F., et al. 2016, MNRAS, 463, 2819

Mieske, S., Hilker, M., \& Infante, L. 2002, A\&A, 383, 823

Mieske, S., Hilker, M., \& Infante, L. 2004, A\&A, 418, 445

Mieske, S., Hilker, M., Jordán, A., et al. 2008, A\&A, 487, 921

Mieske, S., Jordán, A., Côté, P., et al. 2010, ApJ, 710, 1672

Mo, H., van den Bosch, F. C., \& White, S. 2010, Galaxy Formation and Evolution (Cambridge University Press)

Moore, B., Diemand, J., Madau, P., Zemp, M., \& Stadel, J. 2006, MNRAS, 368, 563

Muratov, A. L., \& Gnedin, O. Y. 2010, ApJ, 718, 1266

Ostrov, P., Geisler, D., \& Forte, J. C. 1993, AJ, 105, 1762

Ostrov, P. G., Forte, J. C., \& Geisler, D. 1998, AJ, 116, 2854

Paolillo, M., Fabbiano, G., Peres, G., \& Kim, D.-W. 2002, ApJ, 565, 883

Paolillo, M., Puzia, T. H., Goudfrooij, P., et al. 2011, ApJ, 736, 90

Park, H. S., \& Lee, M. G. 2013, ApJ, 773, L27

Peacock, M. B., Strader, J., Romanowsky, A. J., \& Brodie, J. P. 2015, ApJ, 800, 13

Peng, E. W., Jordán, A., Côté, P., et al. 2006, ApJ, 639, 95

Peng, E. W., Jordán, A., Côté, P., et al. 2008, ApJ, 681, 197

Peng, C. Y., Ho, L. C., Impey, C. D., \& Rix, H.-W. 2010, AJ, 139, 2097

Peng, E. W., Ferguson, H. C., Goudfrooij, P., et al. 2011, ApJ, 730, 23

Piotto, G., Bedin, L. R., Anderson, J., et al. 2007, ApJ, 661, L53

Puzia, T. H., \& Sharina, M. E. 2008, ApJ, 674, 909

Puzia, T. H., Kissler-Patig, M., Thomas, D., et al. 2004, A\&A, 415, 123 
A\&A 611, A93 (2018)

Puzia, T. H., Paolillo, M., Goudfrooij, P., et al. 2014, ApJ, 786, 78

Raimondo, G. 2009, ApJ, 700, 1247

Raimondo, G., Brocato, E., Cantiello, M., \& Capaccioli, M. 2005, AJ, 130, 2625

Richtler, T. 2006, Bulletin of the A. S. of India, 34, 83

Richtler, T., Dirsch, B., Gebhardt, K., et al. 2004, AJ, 127, 2094

Robin, A. C., Reylé, C., Derrière, S., \& Picaud, S. 2003, A\&A, 409, 523

Scharf, C. A., Zurek, D. R., \& Bureau, M. 2005, ApJ, 633, 154

Schlafly, E. F., \& Finkbeiner, D. P. 2011, ApJ, 737, 103

Schlegel, D. J., Finkbeiner, D. P., \& Davis, M. 1998, ApJ, 500, 525

Schuberth, Y., Richtler, T., Bassino, L., \& Hilker, M. 2008, A\&A, 477, L9

Schuberth, Y., Richtler, T., Hilker, M., et al. 2010, A\&A, 513, A52

Smart, R. L., \& Nicastro, L. 2013, VizieR Online Data Catalog: I/324

Spearman, C. 1904, The American Journal of Psychology, 15, 72

Spitler, L. R., \& Forbes, D. A. 2009, MNRAS, 392, L1

Strader, J., Brodie, J. P., Cenarro, A. J., Beasley, M. A., \& Forbes, D. A. 2005, AJ, 130,1315
Strader, J., Beasley, M. A., \& Brodie, J. P. 2007, AJ, 133, 2015 Tonry, J. L., Dressler, A., Blakeslee, J. P., et al. 2001, ApJ, 546, 681 Usher, C., Forbes, D. A., Brodie, J. P., et al. 2012, MNRAS, 426, 1475 VandenBerg, D. A., Brogaard, K., Leaman, R., \& Casagrande, L. 2013, ApJ, 775, 134

Villegas, D., Jordán, A., Peng, E. W., et al. 2010, ApJ, 717, 603 Voggel, K., Hilker, M., \& Richtler, T. 2016, A\&A, 586, A102

Wagner, S., Richtler, T., \& Hopp, U. 1991, A\&A, 241, 399

Wang, Q., Peng, E. W., Blakeslee, J. P., et al. 2013, ApJ, 769, 145

Webb, J. J., Sills, A., Harris, W. E., et al. 2016, MNRAS, 460, 2129

Wittmann, C., Lisker, T., Pasquali, A., Hilker, M., \& Grebel, E. K. 2016, MNRAS, 459, 4450

Woodley, K. A., Harris, W. E., Puzia, T. H., et al. 2010, ApJ, 708, 1335

Yoon, S.-J., Yi, S. K., \& Lee, Y.-W. 2006, Science, 311, 1129

Yoon, S.-J., Lee, S.-Y., Blakeslee, J. P., et al. 2011, ApJ, 743, 150

Yoon, S.-J., Sohn, S. T., Kim, H.-S., et al. 2013, ApJ, 768, 137 


\section{Appendix A: Sources in the fields}

The VST images used for the present study are extremely rich in details. The complete catalog of selected sources in the three bands used is available both via CDS interface, and through the VEGAS survey web pages. Here we report several interesting objects, in particular for the field of NGC 3115.

\section{A.1. Ultra compact dwarfs (UCDs)}

The selection of UCDs was carried out based on the same approach adopted for GCs, i.e., we rely on the observed properties of known UCD to select a sample of candidates. As already discussed in Cantiello et al. (2015), the sample of UCDs present in Jennings et al. (2014) reveals significant contamination from non-UCDs. Hence, we decided to use as reference the sole sample of UCDs from the Fornax cluster sample. As shown in the color-color diagrams in Fig. 3, the UCD candidates from NGC 3115 are highly scattered around the GCs and the galaxies sequences, while the UCDs candidates close to NGC 1399 all spectroscopically confirmed - line up with the GC sequence, with negligible scatter around it.

The evidence shown in Cantiello et al. (2015) that some UCDs selected by Jennings et al. are clearly background objects, together with the large color-scatter around the GC and SSP sequence, and the purely photometric nature of the selection criterion adopted by the authors, motivated our choice to reject the sample of UCDs from Jennings et al. for defining the selection criteria of UCDs. We rather adopted the properties of UCDs in Fornax for selecting UCD candidates around NGC 3115, i.e., we assumed that the expected locus of UCDs in (nearly) all the parameter spaces does not change significantly for the two galaxies. In particular, with respect to the GC selection parameters reported in Table 2, we adopted larger values for the morphological parameters, magnitude limits $M_{g} \geq-10$ mag and $M_{i} \geq-11 \mathrm{mag}$, while the same color selection as for GCs was used for UCDs. No size cut has been applied to UCDs.

For NGC 1399, adopting such criteria calibrated on known UCDs and after rejecting objects too close to bright contaminants (either stars or other galaxies) or to the edges of the frame, we ended up with a selection of 86 candidates. To further clean the sample, we analyzed the azimuthally averaged radial profiles of the 86 UCD candidates (and of their power spectra), comparing these candidates with the profiles of known UCD candidates. The culled sample contained 13 objects. By cross-correlating the sample of 13 objects with available catalogs (NED, VizieR), we obtained that 4 of these objects were observed and spectroscopically classified as foreground stars by Mieske et al. (2002, 2004), and 8 more are UCDs from other catalogs. Hence, the UCD selection adopted has a success rate of $\sim 67 \%$. Only one of the candidates, at RA $(\mathrm{J} 2000)=03 \mathrm{~h} 39 \mathrm{~m} 51.2 \mathrm{~s} \mathrm{Dec}(\mathrm{J} 2000)=$ $-35 \mathrm{~d} 37 \mathrm{~m} 28.6 \mathrm{~s}$, does not match with any previously known

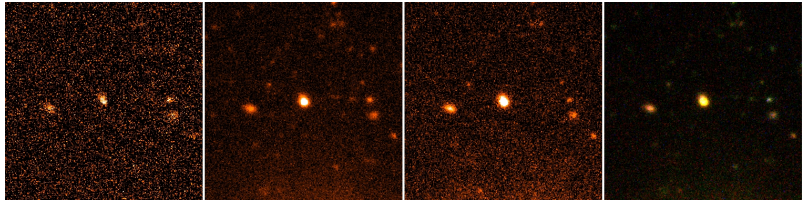

Fig. A.1. From left to right: ugi and RGB thumbnail of the UCD candidate selected in the $\sim 1$ sq. degree area centered on NGC 1399. The obvious asymmetry of the source, especially seen in the $u$ band cutout, is shown. The boxes are $1^{\prime}$ on each side.

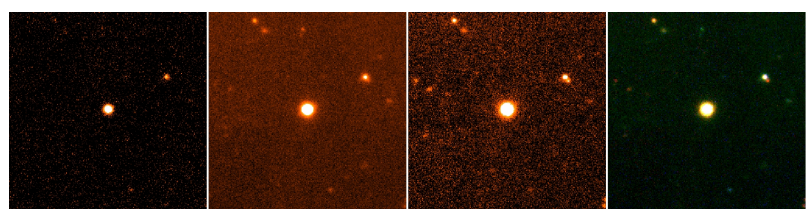

Fig. A.2. From left to right: ugi and RGB thumbnail of one UCD candidate in the $\sim 1$ sq. degree area centered on NGC 3115 . The boxes are $1^{\prime}$ on each side.

extragalactic source, and has measured proper motion compatible with zero within uncertainties (Smart \& Nicastro 2013). The source overlaps with the GALEX Medium Imaging Survey Catalog source GALEXMSC J033951.22-353728.2, with FUV-NUV 0 1.09, which is within the range of observed UV colors for UCDs Mieske et al. (2008), but is also consistent with the locus of MW stars. Figure A.1 shows the ugi and RGB color thumbnail centered on the UCD candidate. Given the significant asymmetry of the source, similar to the cases discussed in Voggel et al. (2016) and Wittmann et al. (2016), and its proper motion consistent with zero, we are inclined to consider it an extragalactic source. However, whether it is a UCD or not cannot be concluded from the present dataset. Spectroscopic observations are needed to definitely classify the source.

For NGC 3115, a sample of 76 UCD candidates was preselected. After inspecting the azimuthal averaged radial profiles (and the profiles of the power spectra) and cross-correlating with available catalogs, the final list of UCD candidates contains 24 objects listed in Table A.1. One of the selected candidates is shown in Fig. A.2.

\section{A.2. Other peculiar objects in the frame of NGC 3115}

We also inspected the VST frame in search of low surface brightness (LSB) objects and other peculiar sources. The coordinates of the selected objects are given in Table A.2. The thumbnail of the objects are reported in the Figs. A.3-A.5. In particular, we show what seems to be a cluster of galaxies with a strong gravitational lens (Fig. A.3), several interacting galaxies (Fig. A.4), some galaxies with rings and bars (Fig. A.5) and, finally, the selected LSB candidates (Fig. A.6). 
Table A.1. Coordinates of UCD candidates in the field of NGC 3115.

\begin{tabular}{lccccc}
\hline \hline ID & RA $(\mathrm{J} 2000)$ & Dec $(\mathrm{J} 2000)$ & $m_{u}(\mathrm{mag})$ & $m_{g}(\mathrm{mag})$ & $m_{i}(\mathrm{mag})$ \\
\hline 1 & 151.35565 & -8.24734 & $20.387 \pm 0.008$ & $19.529 \pm 0.002$ & $19.090 \pm 0.003$ \\
2 & 151.37987 & -8.17344 & $21.257 \pm 0.012$ & $19.572 \pm 0.002$ & $18.654 \pm 0.002$ \\
3 & 151.39560 & -8.15749 & $19.800 \pm 0.005$ & $18.417 \pm 0.001$ & $17.715 \pm 0.001$ \\
4 & 151.40596 & -8.05649 & $19.609 \pm 0.005$ & $18.753 \pm 0.002$ & $18.229 \pm 0.002$ \\
5 & 151.35355 & -7.98409 & $19.577 \pm 0.005$ & $18.397 \pm 0.001$ & $17.643 \pm 0.001$ \\
6 & 151.39183 & -7.98296 & $19.990 \pm 0.006$ & $18.652 \pm 0.002$ & $17.832 \pm 0.002$ \\
7 & 151.58919 & -7.96235 & $20.365 \pm 0.008$ & $19.537 \pm 0.002$ & $19.027 \pm 0.003$ \\
8 & 151.42009 & -7.85778 & $20.337 \pm 0.007$ & $18.705 \pm 0.001$ & $17.739 \pm 0.001$ \\
9 & 151.50733 & -7.78802 & $19.587 \pm 0.005$ & $18.323 \pm 0.001$ & $17.561 \pm 0.001$ \\
10 & 151.00620 & -7.74170 & $20.475 \pm 0.008$ & $19.072 \pm 0.002$ & $18.220 \pm 0.002$ \\
11 & 151.56052 & -7.72850 & $19.382 \pm 0.004$ & $18.141 \pm 0.001$ & $17.469 \pm 0.001$ \\
12 & 151.11798 & -7.70166 & $18.682 \pm 0.003$ & $17.704 \pm 0.001$ & $17.000 \pm 0.001$ \\
13 & 151.55324 & -7.61832 & $20.807 \pm 0.009$ & $19.428 \pm 0.002$ & $18.522 \pm 0.002$ \\
14 & 151.42659 & -7.51542 & $19.863 \pm 0.006$ & $18.866 \pm 0.002$ & $18.355 \pm 0.002$ \\
15 & 151.16905 & -7.47111 & $20.706 \pm 0.010$ & $19.726 \pm 0.003$ & $19.030 \pm 0.003$ \\
16 & 151.40532 & -7.46064 & $20.193 \pm 0.007$ & $18.988 \pm 0.002$ & $18.292 \pm 0.002$ \\
17 & 151.25995 & -7.45713 & $21.118 \pm 0.015$ & $19.587 \pm 0.003$ & $18.797 \pm 0.002$ \\
18 & 151.78131 & -7.37360 & $20.158 \pm 0.007$ & $18.787 \pm 0.002$ & $17.919 \pm 0.002$ \\
19 & 151.77539 & -7.26576 & $21.850 \pm 0.020$ & $19.572 \pm 0.003$ & $18.193 \pm 0.002$ \\
10 & 151.53886 & -7.21417 & $20.742 \pm 0.016$ & $19.837 \pm 0.005$ & $19.233 \pm 0.006$ \\
21 & 151.60184 & -7.83037 & $20.543 \pm 0.008$ & $18.677 \pm 0.001$ & $17.296 \pm 0.001$ \\
22 & 151.25984 & -7.81241 & $19.896 \pm 0.006$ & $18.416 \pm 0.001$ & $17.491 \pm 0.001$ \\
23 & 151.12245 & -7.76488 & $18.353 \pm 0.002$ & $17.404 \pm 0.002$ & $16.547 \pm 0.001$ \\
24 & 151.43657 & -7.76087 & $20.565 \pm 0.008$ & $19.491 \pm 0.002$ & $18.930 \pm 0.003$ \\
\hline
\end{tabular}
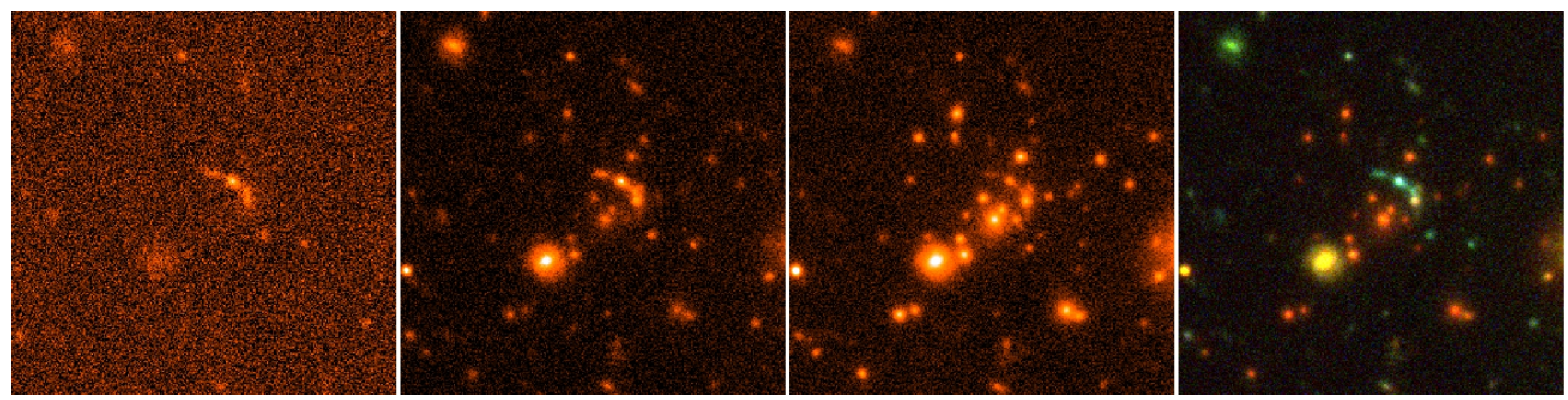

Fig. A.3. From left to right: ugi and RGB thumbnail of a lens candidate. The cutout size is $1^{\prime} \times 1^{\prime}$.

Table A.2. Coordinates of peculiar objects in the field of NGC 3115.

\begin{tabular}{lcc}
\hline \hline ID & RA $(\mathrm{J} 2000)$ & Dec $(\mathrm{J} 2000)$ \\
\hline LSB\#1 & 151.55289 & -7.5001689 \\
LSB\#2 & 151.61118 & -7.5495657 \\
LSB\#3 & 151.6404 & -7.5095855 \\
LSB\#4 & 151.3558 & -7.7283793 \\
LSB\#5 & 151.36609 & -7.7305557 \\
LSB\#6 & 151.29081 & -7.5397223 \\
LSB\#7 & 151.30012 & -7.5492535 \\
LSB\#8 & 151.47394 & -7.8804395 \\
Interacting\#1 & 151.41344 & -7.4736593 \\
Interacting\#2 & 150.78087 & -8.1403071 \\
Interacting\#3 & 150.94656 & -7.4637473 \\
Interacting\#4 & 151.68343 & -7.7048583 \\
Ring\#1 & 150.77724 & -7.4205627 \\
Ring\#2 & 151.30856 & -8.0436752 \\
Ring\#3 & 151.62259 & -7.6092057 \\
Ring\#4 & 151.60656 & -7.5072465 \\
Lens & 151.71811 & -7.9269669 \\
\hline
\end{tabular}


M. Cantiello et al.: VEGAS-SSS: Genetics of globular cluster systems

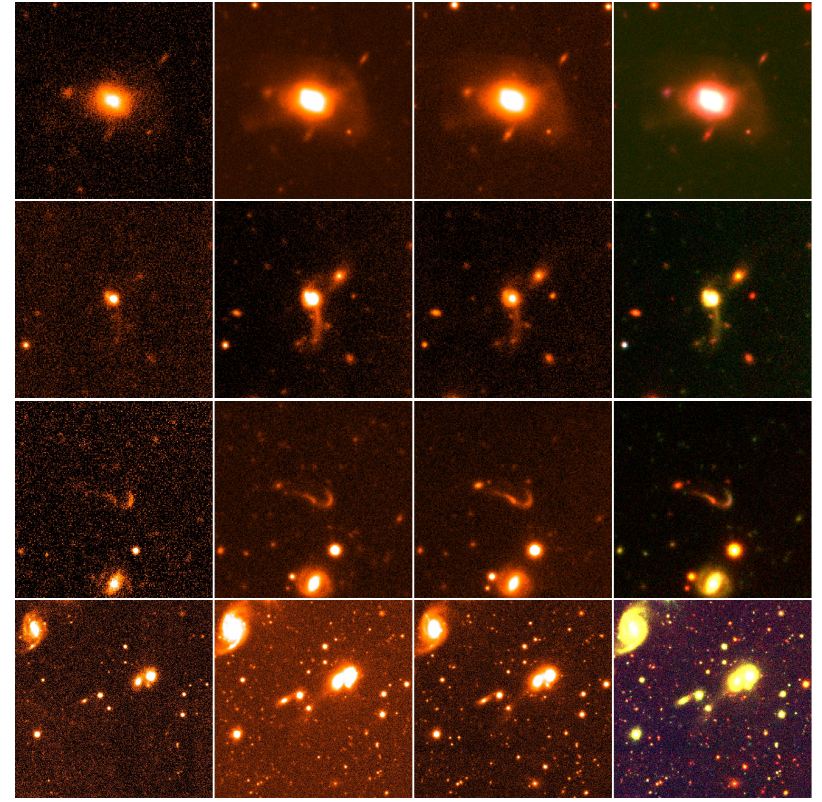

Fig. A.4. From left to right: ugi and RGB thumbnail of interacting candidates from ID\#1 to \#4. All boxes are $1^{\prime} \times 1^{\prime}$, except for the lowermost cutout showing an area of $3^{\prime} \times 3^{\prime}$.

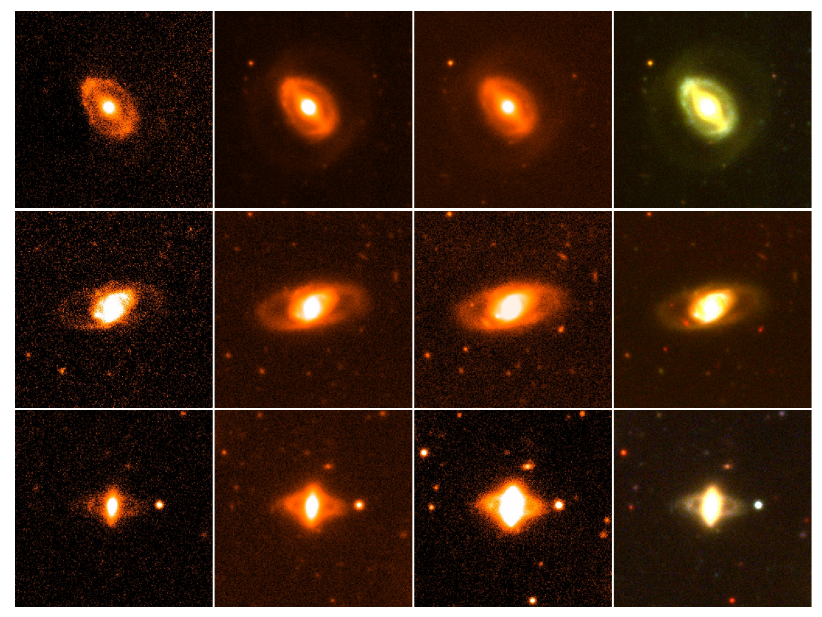

Fig. A.5. From left to right: ugi and RGB thumbnail of ring galaxies candidates from ID\#1 to \#3. The ringed galaxy with ID\#4, close to LSB\#2-LSB\#3 candidates, appears in the second row of thumbnail in Fig. A.6. The cutout size is $1^{\prime} \times 1^{\prime}$.

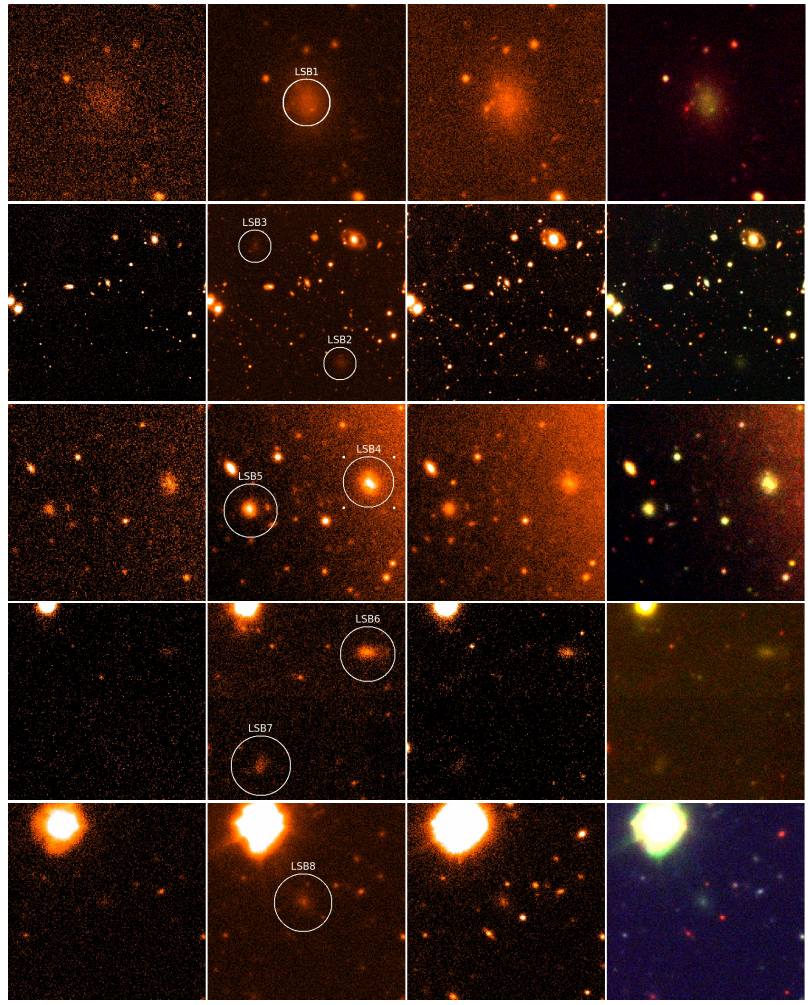

Fig. A.6. From left to right: ugi and RGB thumbnail of LSB candidates. The cutout size is $1^{\prime} \times 1^{\prime}$, except for the second cutout showing an area of $4^{\prime} \times 4^{\prime}$. 